TRANSACTIONS OF THE

AMERICAN MATHEMATICAL SOCIETY

Volume 363, Number 8, August 2011, Pages 4475-4503

S 0002-9947(2011)05418-8

Article electronically published on March 14, 2011

\title{
THE IMAGE OF THE MAP FROM GROUP COHOMOLOGY TO GALOIS COHOMOLOGY
}

\author{
M. TEZUKA AND N. YAGITA
}

\begin{abstract}
We study the image of the natural map from group cohomology to Galois cohomology by using motivic cohomology of classifying spaces.
\end{abstract}

\section{INTRODUCTION}

Let $k$ be a field of $\operatorname{ch}(k)=0$, which contains a $p$-th root of unity. Let $G$ be a split affine algebraic group over $k, V$ a faithful representation of $G$, and $k(V)^{G}$ the invariant field. When $G$ is a finite group, we have the natural quotient map of groups $q: \operatorname{Gal}\left(\bar{k}(\bar{V}) / k(W)^{G}\right) \rightarrow G$, which induces the map from the group cohomology of $G$ to the Galois cohomology of $k(V)^{G}$.

This map is also defined for connected groups (and finite groups) by using the classifying space $B G$ defined by Totaro and Voevodsky ([To], [Vo4]). Given degree $*>1$, let $W_{*}$ be a faithful representation of $G$ such that $G$ acts freely on $W_{*}-S_{*}$ for some closed subset $S_{*}$ with $\operatorname{codim}_{W_{*}} S_{*}>*$, e.g., $W_{*}=V^{\oplus(*+1)}$. Then we can define the map (see $\S 3$ below)

$$
q^{*}: H^{*}(B G ; \mathbb{Z} / p) \rightarrow H^{*}\left(k\left(W_{*}\right)^{G} ; \mathbb{Z} / p\right) .
$$

The purpose of this paper is to study the image $\operatorname{Im}\left(q^{*}\right)$ by using the motivic cohomology defined by Suslin and Voevodsky [Vo1, Vo2, [Vo3]. The image $\operatorname{Im}\left(q^{*}\right)$ is called the stable cohomology in [Bo, [Bo-Pe-Ts]. The kernel $\operatorname{Ker}\left(q^{*}\right)=N g(G)$ is called the negligible ideal $\mathrm{Pe}$, $\mathrm{Sa}$.

Let $H^{*, *^{\prime}}(X ; \mathbb{Z} / p)$ be the $\bmod (p)$ motivic cohomology. Let

$$
0 \neq \tau \in H^{0,1}(\operatorname{Spec}(k) ; \mathbb{Z} / p) \cong \mathbb{Z} / p .
$$

Using an affirmative solution of the Bloch-Kato conjecture by Voevodsky (and hence the Beilinson-Lichtenbaum conjecture), the map $q^{*}$ is decomposed as

$$
q^{*}: H^{*, *}(B G ; \mathbb{Z} / p) \rightarrow H^{*, *}(B G ; \mathbb{Z} / p) /(\tau) \rightarrow H^{*, *}\left(\operatorname{Spec}\left(k\left(W_{*}\right)^{G}\right) ; \mathbb{Z} / p\right),
$$

where $H^{*, *}(B G ; \mathbb{Z} / p) /(\tau)=H^{*, *}(B G ; \mathbb{Z} / p) /\left(\tau H^{*, *-1}(B G ; \mathbb{Z} / p)\right)$.

By the Belinson-Lichtenbaum conjecture and the work of Bloch-Ogus Bl-Og, we know that

$$
H^{*, *}(B G ; \mathbb{Z} / p) /(\tau) \subset H_{Z a r}^{0}\left(B G ; H_{\mathbb{Z} / p}^{*}\right) \subset H^{*}\left(k\left(W_{*}\right)^{G} ; \mathbb{Z} / p\right) .
$$

Received by the editors April 22, 2009 and, in revised form, February 11, 2010 and June 3, 2010

2010 Mathematics Subject Classification. Primary 11E72, 12G05; Secondary 55R35.

Key words and phrases. Cohomolgical invariant, classifying spaces, motivic cohomology.

(C)2011 American Mathematical Society 
Here $H_{\mathbb{Z} / p}^{*}$ is the Zariski sheaf induced from the presheaf $H_{e t}^{*}(V ; \mathbb{Z} / p)$ for an open subset $V$ of $B G$. Therefore we see $\mathrm{Or}-\mathrm{Vi}-\mathrm{Vo}$

$$
\operatorname{Im}\left(q^{*}\right)=H^{*}(B G ; \mathbb{Z} / p) /(N g) \cong H^{*, *}(B G ; \mathbb{Z} / p) /(\tau) .
$$

Note that the ring on the right-hand side does not depend on the choice of $W_{*}$. We also note that the ideal $(N g)=N g(G)$ coincides with the coniveau filtration $N^{1} H^{*}(B G ; \mathbb{Z} / p)$ defined by Grothendieck.

In this paper we compute $\operatorname{Im}\left(q^{*}\right)$ (when $k$ is an algebraically closed field in $\mathbb{C}$ ) for abelian $p$-groups, $O_{n}, S O_{n}, \operatorname{Spin}_{n}, P G L_{p}$, and exceptional groups. Extraspecial $p$-groups are also studied. For example, we see

$$
H^{*}\left(\operatorname{BSpin}_{n} ; \mathbb{Z} / 2\right) /(N g) \cong \mathbb{Z} / 2 \text { for } n \geq 3 .
$$

Recall that the cohomological invariant $\operatorname{Inv}(G ; \mathbb{Z} / p)$ is (roughly speaking) a ring of natural maps $H^{1}(F ; G) \rightarrow H^{*}(F ; \mathbb{Z} / p)$ for finitely generated fields $F$ over $k$. This ring is very well studied; for example, see Ga-Me-Se. In particular, it is very useful to compute the essential dimension ed $(G)$ of $G$ ([Re], [Br-Re-Vi] $)$. Moreover, Totaro proved that

$$
\operatorname{Inv}^{*}(G ; \mathbb{Z} / p) \cong H_{Z a r}^{0}\left(B G ; H_{\mathbb{Z} / p}^{*}\right)
$$

in a letter to Serre Ga-Me-Se]. Hence $\operatorname{Im}\left(q^{*}\right) \subset \operatorname{Inv} v^{*}(G ; \mathbb{Z} / p)$.

We use these results for some parts of this paper. However, we also give new explanations of $\operatorname{Inv}^{*}(G ; \mathbb{Z} / p)$ for the case that $k$ is an algebraically closed field in $\mathbb{C}$. For example, the image of a (topological) Stiefel-Whitney class $w_{i}$ of the map

$$
H^{*}\left(B O_{n} ; \mathbb{Z} / 2\right) \rightarrow H^{*}\left(B O_{n} ; \mathbb{Z} / 2\right) /(N g) \subset \operatorname{Inv}^{*}\left(O_{n} ; \mathbb{Z} / 2\right)
$$

is indeed the Stiefel-Whitney class $w_{i}$ defined by Milnor and Serre as the natural function from quadratic forms to Milnor $K$-theories.

All examples stated above are detected by abelian $p$-subgroups $A$ of $G$, i.e., the restriction map

$$
\text { Res : } H^{*}(B G ; \mathbb{Z} / p) /(N g) \rightarrow \Pi_{A} H^{*}(B A ; \mathbb{Z} / p) /(N g)
$$

is injective. (Indeed, most of the above cases are detected by only one elementary abelian $p$-subgroup.)

Of course, this detected property does not hold for general $G$. However, to give examples is not so easy. Indeed, for a $p$-group $G$ of exponent $p$, if $H^{2}(B G ; \mathbb{Z} / p) /(N g)$ is not detected by any $A \cong \mathbb{Z} / p \oplus \mathbb{Z} / p$, then $G$ is a counterexample of Noether's problem, namely, $k\left(W_{2}\right)^{G}$ is not purely transcendental over $k$. The examples of Saltman and Bogomolov are essentially of these types [Sa].

For each $n>1$, we give an example $G_{n}$ of a $p$-group $p \geq 3$, such that $H^{2 n}\left(G_{n} ; \mathbb{Z} / p\right)$ is not detected by abelian $p$-subgroups, while it does not imply a counterexample of Noether's problem. Here the composition $Q_{2 n-2} \ldots Q_{0}(x)$ of Milnor operations is used to see $x \notin N g(G)$ given $x \in H^{2 n}(B G ; \mathbb{Z} / p)$.

An outline of this paper is the following. In $\S 2$, we recall the properties of the motivic cohomology and coniveau filtrations. In $\S 3$, we study cohomology of groups $H^{*}(B G ; \mathbb{Z} / p)$ and the definitions of stable cohomology and negligible ideals $N g(G)$. In particular, we give conditions for $x \in N g(G)$ by using cohomology operations. In $\S 4$, we recall the definition of the cohomological invariants. In $\S \S 5-8$, we study cohomological invariants for concrete groups, e.g., abelian $p$-groups, $O_{n}$, $S O_{n}, S_{p i n}$, exceptional groups, and $P G L_{p}$. Extraspecial $p$-groups are studied in $\S 9$ and are also used in $\S \S 11-12$. We recall Noether's problem in $\S 10$. Saltman's 
example (which is a special $p$-group) is studied in $\S 11$. In the last section, we give an example for the above non-detected problem.

\section{Motivic Cohomology}

Let $X$ be a smooth (quasi-projective) variety. Let $H^{*, *^{\prime}}(X ; \mathbb{Z} / p)$ be the $\bmod (p)$ motivic cohomology defined by Voevodsky and Suslin.

Recall that the $(\bmod p) B L(n, p)$ condition holds if

$$
H^{m, n}(X ; \mathbb{Z} / p) \cong H_{e t}^{m}\left(X ; \mu_{p}^{\otimes n}\right) \quad \text { for all } m \leq n .
$$

It is known that the $B L(n, p)$ condition holds for $p=2$ or $n=2$ by Voevodsky [Vo1, Vo2, and Merkurjev-Suslin, respectively. Recently M. Rost and V. Voevodsky ( $\mathrm{Vo5}$, $\mathrm{Su}-\mathrm{Jo}, \mathrm{Ro}$ ) proved that the $B L(n, p)$ condition holds for each $p$ and $n$. Hence the Bloch-Kato conjecture also holds. Therefore, in this paper, we always assume the $B L(n, p)$-condition and also the Bloch-Kato conjecture for all $n, p$.

Moreover, we always assume that $k$ contains a primitive $p$-th root of unity. For these cases, we see the isomorphism $H_{e t}^{m}\left(X ; \mu_{p}^{\otimes n}\right) \cong H_{e t}^{m}(X ; \mathbb{Z} / p)$. Let $\tau$ be a generator of $H^{0,1}(\operatorname{Spec}(k) ; \mathbb{Z} / p) \cong \mathbb{Z} / p$, so that

$$
\operatorname{colim}_{i} \tau^{i} H^{*, *^{\prime}}(X ; \mathbb{Z} / p) \cong H_{e t}^{*}(X ; \mathbb{Z} / p) .
$$

Recall that $\mathbb{Z} / p(n)[\mathrm{Vo1}$, , Vo2, $\mathrm{Vo3}$ is the complex of sheaves in Zariski topology such that $H^{m, n}(X ; \mathbb{Z} / p) \cong H_{Z a r}^{m}(X ; \mathbb{Z} / p(n))$. Let $\alpha$ be the obvious map of sites from etale topology to Zariski topology so that $\alpha^{*} \mathbb{Z} / p(1) \cong \mu_{p}$ of etale sheaves and hence

$$
H_{e t}^{m}(X ; \mathbb{Z} / p) \cong H_{e t}^{m}\left(X ; \mu_{p}^{\otimes n}\right) \cong H_{Z a r}^{m}\left(X, R \alpha_{*} \alpha^{*} \mathbb{Z} / p(n)\right) .
$$

For $k \leq n$, let $\tau_{\leq k} R \alpha_{*} \alpha^{*} \mathbb{Z} / p(n)$ be the canonical truncation of $R \alpha_{*} \alpha^{*} \mathbb{Z} / p(n)$ of level $k$. Then we have the exact triangle in the derived category of Zariski sheaves

$$
\rightarrow \tau_{\leq n-1} R \alpha_{*} \alpha^{*} \mathbb{Z} / p(n) \rightarrow \tau_{\leq n} R \alpha_{*} \alpha^{*} \mathbb{Z} / p(n) \rightarrow H_{\mathbb{Z} / p}^{n}[-n] \rightarrow,
$$

where $H_{\mathbb{Z} / p}^{n}$ is the Zariski sheaf induced from the presheaf $H_{e t}^{n}(V ; \mathbb{Z} / p)$ for an open subset $V$ of $X$. The Beilinson and Lichtenbaum conjecture (hence the $B L(n, p)$ condition) (see [Vo2, [Vo5]) implies

$$
\mathbb{Z} / p(k) \cong \tau_{\leq k} R \alpha_{*} \alpha^{*} \mathbb{Z} / p(n) .
$$

Hence we have

Lemma 2.1 ( $\mathrm{Or}-\mathrm{Vi}-\mathrm{Vo}, \overline{\mathrm{Vo5}}])$. There is a long exact sequence

$$
\begin{aligned}
\rightarrow H^{m, n-1}(X ; \mathbb{Z} / p) & \stackrel{\times \tau}{\rightarrow} H^{m, n}(X ; \mathbb{Z} / p) \\
& \rightarrow H_{Z \text { Zar }}^{m-n}\left(X ; H_{\mathbb{Z} / p}^{n}\right) \rightarrow H^{m+1, n-1}(X ; \mathbb{Z} / p) \stackrel{\times \tau}{\rightarrow} .
\end{aligned}
$$

In particular, we have

Corollary 2.2. There is a short exact sequence

$$
0 \rightarrow H^{m, n}(X ; \mathbb{Z} / p) /(\tau) \rightarrow H_{Z a r}^{m-n}\left(X ; H_{\mathbb{Z} / p}^{n}\right) \rightarrow{ }_{\tau} H^{m+1, n-1}(X ; \mathbb{Z} / p) \rightarrow 0,
$$

where $H^{m, n}(X ; \mathbb{Z} / p) /(\tau)=H^{m, n}(X ; \mathbb{Z} / p) /\left(\tau H^{m, n-1}(X ; \mathbb{Z} / p)\right)$ and

$$
{ }_{\tau} H^{m+1, n-1}(X ; \mathbb{Z} / p)=\operatorname{Ker}(\tau) \mid H^{m+1, n-1}(X ; \mathbb{Z} / p) .
$$


Note that the above long exact sequence induces the $\tau$-Bockstein spectral sequence

$$
E(\tau)_{1}^{m, n}=H_{Z a r}^{m-n}\left(X ; H_{\mathbb{Z} / p}^{n}\right) \Longrightarrow \operatorname{colim}_{i} \tau^{i} H^{m, n}(X ; \mathbb{Z} / p) \cong H_{e t}^{m}(X ; \mathbb{Z} / p) .
$$

On the other hand, the filtration coniveau is given by

$$
N^{c} H_{e t}^{m}(X ; \mathbb{Z} / p)=\bigcup_{Z} K e r\left\{H_{e t}^{m}(X ; \mathbb{Z} / p) \rightarrow H_{e t}^{m}(X-Z ; \mathbb{Z} / p)\right\},
$$

where $Z$ runs in the set of closed subschemes of $X$ of codim $=c$. Grothendieck wrote the $E_{1}$-term of the spectral sequence induced from the above coniveau filtration,

$$
E(c)_{1}^{c, m-c} \cong \Pi_{x \in X^{(c)}} H_{e t}^{m-2 c}(k(x) ; \mathbb{Z} / p) \Longrightarrow H_{e t}^{m}(X ; \mathbb{Z} / p),
$$

where $X^{(c)}$ is the set of primes of codimension $c$ and $k(x)$ is the residue field of $x$. We can regard $i_{x *} H_{e t}^{m-2 c}(k(x) ; \mathbb{Z} / p)$ as a constant sheaf $H_{e t}^{m-2 c}(k(x) ; \mathbb{Z} / p)$ on $\{\bar{x}\}$ and extend it by zero to $X$. Then the differentials of the spectral sequence give us a complex on sheaves on $X$ :

$$
\begin{aligned}
0 \rightarrow H_{\mathbb{Z} / p}^{q} & \rightarrow \Pi_{x \in X^{(0)}} i_{x *} H_{e t}^{q}(k(x) ; \mathbb{Z} / p) \rightarrow \Pi_{x \in X^{(1)}} i_{x *} H_{e t}^{q-1}(k(x) ; \mathbb{Z} / p) \\
& \rightarrow \cdots \rightarrow \Pi_{x \in X^{(q)}} i_{x *} H_{e t}^{0}(k(x) ; \mathbb{Z} / p) \rightarrow 0 .
\end{aligned}
$$

Bloch-Ogus Bl-Og proved that the above sequence of sheaves is exact and the $E_{2}$-term is given by

$$
E(c)_{2}^{c, m-c} \cong H_{Z a r}^{c}\left(X, H_{\mathbb{Z} / p}^{m-c}\right) .
$$

In particular, we have

\section{Corollary 2.3.}

$$
H_{Z a r}^{0}\left(X ; H_{\mathbb{Z} / p}^{m}\right) \cong \operatorname{Ker}\left\{H_{e t}^{m}(k(X) ; \mathbb{Z} / p) \rightarrow \Pi_{x \in X^{(1)}} H_{e t}^{m-1}(k(x) ; \mathbb{Z} / p)\right\} .
$$

By Deligne (footnote (1) in Remark 6.4 in $\overline{\mathrm{Bl}-\mathrm{Og}}$ ) and Paranjape (Corollary 4.4 in $(\mathrm{Pa}]$ ), it is proven that there is an isomorphism of the coniveau spectral sequence with the Leray spectral sequence for the map $\alpha$. Hence we have

Theorem 2.4 (Deligne, Parajape). There is the isomorphism

$$
E(c)_{r}^{c, m-c} \cong E(\tau)_{r-1}^{m, m-c}
$$

for $r \geq 2$ of spectral sequences. Hence the filtrations are the same for

$$
N^{c} H_{e t}^{m}(X ; \mathbb{Z} / p)=F_{\tau}^{m, m-c},
$$

where

$$
F_{\tau}^{m, m-c}=\operatorname{Im}\left(\times \tau^{c}: H^{m \cdot m-c}(X ; \mathbb{Z} / p) \rightarrow H^{m, m}(X ; \mathbb{Z} / p)\right) .
$$

\section{Cohomology OF GRoups}

Let $G$ be a reductive algebraic group over $k$ acting on an affine variety $W$. A point $x \in W$ is called stable if the orbit $G x$ is closed and the stabilizer group $\operatorname{Stab}(x)$ is a finite group. Let us write by $X^{s}$ the set of stable points in $X$. Then $X^{s}$ is an open subset of $X$ and there is the commutative diagram

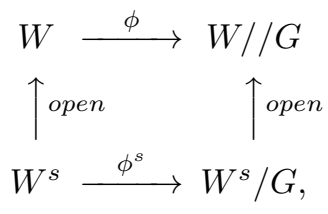


where $W / / G=\operatorname{Spec}\left(k[W]^{G}\right)$, the geometric quotient of $X$. We also note that the invariant field $k(W)^{G}$ is a quotient field of $k[W]^{G}$ when $W^{s} \neq \emptyset$.

Suppose $W^{s} \neq \emptyset$. Then $H^{*, *^{\prime}}(W / / G ; \mathbb{Z} / p)=H^{*, *^{\prime}}\left(\operatorname{Spec}\left(k[W]^{G}\right) ; \mathbb{Z} / p\right)$ and $k\left(W^{s} / G\right)=k(W / / G)=k(W)^{G}$. Hence we have the diagram

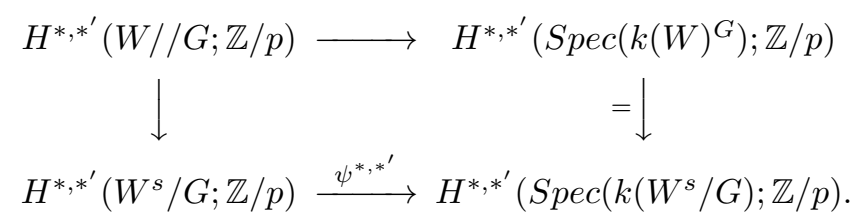

We recall the definition of $B G$ according to Voevodsky and Totaro ( $\S 6$ in Vo4, [To]). For a smooth embedding $X \subset Y$ of codimension $c$, we have the Gysin exact sequence [Vo1, Vo2], Vo3]

$$
\rightarrow H^{*-2 c, *^{\prime}-c}(X ; \mathbb{Z} / p) \stackrel{g_{*}}{\rightarrow} H^{*, *^{\prime}}(Y ; \mathbb{Z} / p) \rightarrow H^{*, *^{\prime}}(Y-X ; \mathbb{Z} / p) \rightarrow .
$$

Since $H^{*,<0}(X ; \mathbb{Z} / p)=0$, we have the isomorphism

$$
H^{*, *^{\prime}}(Y ; \mathbb{Z} / p) \cong H^{*, *^{\prime}}(Y-X ; \mathbb{Z} / p) \quad \text { if } *^{\prime}<c .
$$

The above isomorphism also holds for non-smooth $X$ (e.g., (3.5) in Vo4).

For $n>1$, let $W_{n}$ be a faithful representation of $G$ such that $G$ acts freely on $W_{n}-S_{n}$ for some closed subset $S_{n}$ with $\operatorname{codim}_{W_{n}} S_{n}>n$.

Let us write $U_{n}=\left(W_{n}-S_{n}\right)$ simply. Let $U_{n}^{\prime}=W_{n}^{\prime}-S_{n}^{\prime}$ be another such set. Then we can consider the maps

$$
U_{n} \times_{G} W_{n}^{\prime} \leftarrow U_{n} \times_{G} U_{n}^{\prime} \rightarrow W_{n} \times_{G} U_{n}^{\prime}
$$

which imply the isomorphisms of motivic cohomology for $*^{\prime} \leq n$ from the above result by the Gysin sequence

$$
H^{*, *^{\prime}}\left(U_{n} / G ; \mathbb{Z} / p\right) \cong H^{*, *^{\prime}}\left(U_{n} \times_{G} U_{n}^{\prime} ; \mathbb{Z} / p\right) \cong H^{*, *^{\prime}}\left(U_{n}^{\prime} / G ; \mathbb{Z} / p\right) .
$$

Hence the motivic cohomology $H^{*, *^{\prime}}\left(U_{n} / G ; \mathbb{Z} / p\right)$ for $*^{\prime} \leq n$ does not depend on the choice of such $\left(W_{n}, S_{n}\right)$ (Proposition 6.1 in [Vo4]).

Let $V$ be a faithful representation. Then we can take $W_{n}=V^{\oplus(n+1)}$, for example. The classifying space $B G$ of $G$ is defined as $\operatorname{colim}_{n \rightarrow \infty}\left(U_{n} / G\right)$. The motivic cohomology of $B G$ is given by (Corollary 6.2 in Vo4])

$$
H^{*, *^{\prime}}(B G ; \mathbb{Z} / p) \cong \lim _{n \rightarrow \infty} H^{*, *^{\prime}}\left(U_{n} / G ; \mathbb{Z} / p\right) .
$$

Thus from the above diagram (taking $W=W_{*^{\prime}}$ and $U_{*^{\prime}} \subset W^{s}$ ), we have the map

$$
\begin{aligned}
& \psi^{*, *^{\prime}}: H^{*, *^{\prime}}(B G ; \mathbb{Z} / p) \cong H^{*, *^{\prime}}\left(U_{*^{\prime}} / G ; \mathbb{Z} / p\right) \\
& \quad \rightarrow \quad H^{*, *^{\prime}}\left(\operatorname{Spec}\left(k\left(U_{*^{\prime}} / G\right)\right) ; \mathbb{Z} / p\right) \cong H^{*, *^{\prime}}\left(\operatorname{Spec}\left(k\left(W_{*^{\prime}}\right)^{G}\right) ; \mathbb{Z} / p\right) .
\end{aligned}
$$

In particular, by the $B L(p, *)$ condition, letting $*=*^{\prime}$ we have the map

$$
\psi_{e t}^{*}: H_{e t}^{*}(B G ; \mathbb{Z} / p) \rightarrow H^{*}\left(k\left(W_{*}\right)^{G} ; \mathbb{Z} / p\right) .
$$

When $G$ is a finite group, $H_{e t}^{*}(B G ; \mathbb{Z} / p)$ is isomorphic to the group cohomology $H^{*}(G ; \mathbb{Z} / p)$. Let $\Gamma=\operatorname{Gal}\left(\bar{k}(\bar{V}) / k(V)^{G}\right)$ be the absolute Galois group of the field $k(V)^{G}$. Then the group $G=\operatorname{Gal}\left(k(V) / k(V)^{G}\right)$ is a quotient group of this absolute 
Galois group $\Gamma$. Then the map $\psi_{e t}^{*}$ induces the map $q^{*}$ from the quotient $q: \Gamma \rightarrow G$, i.e.,

$$
\begin{gathered}
q^{*}: H^{*}(G ; \mathbb{Z} / p)=H_{e t}^{*}\left(U_{*} / G ; \mathbb{Z} / p\right) \\
\stackrel{\psi_{e t}^{*}}{\rightarrow} H^{*}\left(k\left(U_{*} / G\right) ; \mathbb{Z} / p\right) \cong H^{*}\left(k\left(W_{*}\right)^{G} ; \mathbb{Z} / p\right) \rightarrow H^{*}\left(k(V)^{G} ; \mathbb{Z} / p\right)=H^{*}(\Gamma ; \mathbb{Z} / p)
\end{gathered}
$$

for $V \subset W_{*}$, e.g., $W_{*}=V^{\oplus(*+1)}$. Of course, we can identify $q^{*}=\psi_{e t}^{*}$ if we take $V$ by $W_{*}$ (i.e., there is such an $S \subset V$ with $\operatorname{codim}_{V}(S)>*$ ).

Lemma 3.1. We have the isomorphism

$$
\operatorname{Im}\left(\psi_{\text {et }}^{*}\right) \cong H^{*, *}(B G ; \mathbb{Z} / p) /(\tau) .
$$

Proof. The composition

$$
H^{*, *}(X ; \mathbb{Z} / p) \rightarrow H_{Z a r}^{0}\left(X ; H_{\mathbb{Z} / p}^{*}\right) \rightarrow H^{*}(k(X) ; \mathbb{Z} / p)
$$

is the restriction map for $\operatorname{Spec}(k(X)) \rightarrow X$ from the following commutative diagram:

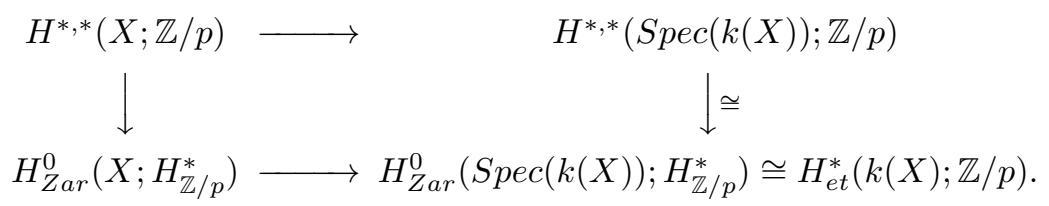

Hence the above restriction map $\psi_{e t}^{*}$ is written as

$$
\begin{aligned}
& \psi^{*, *}: H^{*, *}(B G ; \mathbb{Z} / p) \cong H^{*, *}\left(U_{*} / G ; \mathbb{Z} / p\right) \\
\rightarrow & H_{Z a r}^{0}\left(U_{*} / G ; H_{\mathbb{Z} / p}^{*}\right) \rightarrow H^{*}\left(k\left(W_{*}\right)^{G} ; \mathbb{Z} / p\right) .
\end{aligned}
$$

For each field $F$, by the Bloch-Kato conjecture $H^{*}(F ; \mathbb{Z} / p)$ is multiplicatively generated by elements in $H_{\text {et }}^{1}(F ; \mathbb{Z} / p) \cong H^{1,1}(\operatorname{Spec}(F) ; \mathbb{Z} / p)$. So

$$
\psi^{*, *-1}: H^{*, *-1}(B G ; \mathbb{Z} / p) \rightarrow H^{*, *-1}\left(\operatorname{Spec}\left(k\left(W_{*}\right)^{G}\right) ; \mathbb{Z} / p\right)=0 .
$$

Hence the map $\psi^{*, *}$ is expressed as a composition

$$
H^{*, *}(B G ; \mathbb{Z} / p) \rightarrow H^{*, *}(B G ; \mathbb{Z} / p) /(\tau) \rightarrow H^{*}\left(k\left(W_{*}\right)^{G} ; \mathbb{Z} / p\right) .
$$

The first map is of course surjective, and we only need the injectivity of the second map. Indeed, from Corollaries 2.2 and 2.3, we see that

$$
\begin{aligned}
& H^{*, *}(B G ; \mathbb{Z} / p) /(\tau) \cong H^{*, *}\left(U_{*} / G ; \mathbb{Z} / p\right) /(\tau) \\
& \quad \subset H_{Z a r}^{0}\left(U_{*} / G ; H_{\mathbb{Z} / p}^{*}\right) \subset H^{*}\left(k\left(W_{*}\right)^{G} ; \mathbb{Z} / p\right) .
\end{aligned}
$$

Remark. Let us write $\lim _{n \rightarrow \infty} k\left(W_{n}\right)^{G}=k(B G)$ and

$$
H^{*, *^{\prime}}(k(B G) ; \mathbb{Z} / p)=\lim _{n \rightarrow \infty} H^{*, *^{\prime}}\left(\operatorname{Spec}\left(k\left(W_{n}\right)^{G}\right) ; \mathbb{Z} / p\right) .
$$

Moreover, let

$$
H^{*}\left(B G ; H_{\mathbb{Z} / p}^{*^{\prime}}\right)=\lim _{n \rightarrow \infty} H^{*}\left(U_{n} / G, H_{\mathbb{Z} / p}^{*^{\prime}}\right) .
$$

Then we have the injections (by taking limits) of graded rings

$$
\psi_{B G}^{*, *}: H^{*, *}(B G ; \mathbb{Z} / p) /(\tau) \subset H_{Z a r}^{0}\left(B G ; H_{\mathbb{Z} / p}^{*}\right) \subset H^{*}(k(B G) ; \mathbb{Z} / p) .
$$

Almost all arguments about $H^{*}\left(k\left(W_{*}\right)^{G} ; \mathbb{Z} / p\right)$ also hold if we take $H^{*}(k(B G) ; \mathbb{Z} / p)$ instead. 
Recall the coniveau filtration given in $\S 2$ :

$$
N^{c} H_{e t}^{m}(X ; \mathbb{Z} / p)=\bigcup_{Z} K e r\left\{H_{e t}^{m}(X ; \mathbb{Z} / p) \rightarrow H_{e t}^{m}(X-Z ; \mathbb{Z} / p)\right\},
$$

where $Z$ runs in the set of closed subschemes of $X$ of codim $=c$. From Theorem 2.4 , we see

Corollary 3.2. $\operatorname{Im}\left(\psi_{e t}^{*}\right) \cong H_{e t}^{*}(B G ; \mathbb{Z} / p) /\left(N^{1} H_{e t}^{*}(B G ; \mathbb{Z} / p)\right)$.

We say an element $x \in H_{e t}^{*}(B G ; \mathbb{Z} / p)$ is (universal) negligible if $\psi_{e t}^{*}(x)=0$. Let us write the ideal generated by negligible elements by

$$
\operatorname{Ker}\left(\psi^{*}\right)=(N g)=N g(G) \text {. }
$$

Remark. Originally, Serre defines the negligible class $N g(G)_{S}$ as follows. Let $K$ be a field over $k$ and $\Gamma_{K}=\operatorname{Gal}(\bar{K} / K)$. The class $N g(G)_{S}$ is defined as an ideal generated by elements $x \in H^{*}(B G ; \mathbb{Z} / p)$ such that $\psi_{K}^{*}(x)=0$ for all $\psi_{K}: \Gamma_{K} \rightarrow G$. In general, $N g(G) \neq N g(G)_{S}$, but we use only $N g(G)$ as a negligible class in this paper.

From the above lemma, it is immediate that

$$
\begin{gathered}
N g(G)=N^{1} H_{e t}^{*}(B G ; \mathbb{Z} / p) \\
\cong \operatorname{Im}\left(\times \tau \mid H^{*, *-1}(B G ; \mathbb{Z} / p) \rightarrow H^{*, *}(B G ; \mathbb{Z} / p)\right),
\end{gathered}
$$

and we have

$$
\operatorname{Im}\left(\psi_{e t}^{*}\right) \cong H^{*}(B G ; \mathbb{Z} / p) /(N g) \cong H^{*}(B G ; \mathbb{Z} / p) /\left(N^{1}\right) \cong H^{*, *}(B G ; \mathbb{Z} / p) /(\tau) .
$$

The Chow ring $C H^{*}(X) / p$ is identified with the motivic cohomology $H^{2 *, *}(X ; \mathbb{Z} / p)$ Vo1, Vo2, Vo3. The cycle map is written as

$$
c l: C H^{*}(X) / p=H^{2 *, *}(X ; \mathbb{Z} / p) \stackrel{\times \tau^{*}}{\rightarrow} H^{2 *, 2 *}(X ; \mathbb{Z} / p) \cong H_{e t}^{2 *}(X ; \mathbb{Z} / p) .
$$

Hence we see

Lemma 3.3. $\operatorname{Im}\left(\operatorname{cl}\left(\mathrm{CH}^{+}(B G) / p\right)\right) \subset N g(G)$.

Next consider the relation between cohomology operation and the ideal $N g(G)$. Let $t_{\mathbb{C}}: H^{*, *^{\prime}}(X ; \mathbb{Z} / p) \rightarrow H^{*}(X(\mathbb{C}) ; \mathbb{Z} / p)$ be the realization map Vo1] for the inclusion $k \subset \mathbb{C}$. The motivic cohomology has (Bockstein, reduced powered) cohomology operations Vo2, [Vo4]

$$
\begin{gathered}
\beta: H^{*, *^{\prime}}(X ; \mathbb{Z} / p) \rightarrow H^{*+1, *^{\prime}}(X ; \mathbb{Z} / p), \\
P^{i}: H^{*, *^{\prime}}(X ; \mathbb{Z} / p) \rightarrow H^{*+2 i(p-1), *^{\prime}+i(p-1)}(X ; \mathbb{Z} / p),
\end{gathered}
$$

which are compatible with the usual (topological) cohomology operations by the realization map $t_{\mathbb{C}}$. Voevodsky defines the Milnor operation $Q_{i}$ also in the $\bmod p$ motivic cohomology

$$
Q_{i}: H^{*, *^{\prime}}(X ; \mathbb{Z} / p) \rightarrow H^{*+2 p^{i}-1, *^{\prime}+p^{i}-1}(X ; \mathbb{Z} / p) .
$$

Here we define the weight degree by

$$
w(x)=2 n-m\left(\text { resp. }=n^{\prime}-m^{\prime}\right)
$$

for $0 \neq x \in H^{m, n}(X ; \mathbb{Z} / p)$ (resp. $\left.H^{m^{\prime}}\left(X ; H_{\mathbb{Z} / p}^{n^{\prime}}\right)\right)$. Note that $w(x) \geq 0$ for any smooth $X$. (In fact, $H^{*, *^{\prime}}(X ; \mathbb{Z} / p)=0$ for $*>2 *^{\prime}$ and smooth $X$. ) Similarly, we 
also define the weight degree for cohomology operations, e.g., $w(\tau)=2, w\left(P^{i}\right)=0$, $w\left(Q_{j}\right)=-1$.

Let $\rho_{p}=\left(\xi_{p}\right) \in H^{1,1}(\operatorname{Spec}(k) ; \mathbb{Z} / p)=k^{*} /\left(k^{*}\right)^{p}$, where $\xi_{p}$ is the primitive $p$-th root of unity. The $Q_{i}$ operation has the same property as the topological case with $\bmod \left(\rho_{2}\right)$. For example, $Q_{i}$ is a derivation only with $\bmod \left(\rho_{2}\right)$.

Let $A_{p}$ be the $\bmod p$ Steenrod algebra generated by all cohomology operations on $H^{*, *^{\prime}}(X ; \mathbb{Z} / p)$. (Voevodsky proved [Vo6] that $A_{p}$ is multiplicatively generated by elements in $H^{*, *^{\prime}}(\operatorname{Spec}(k) ; \mathbb{Z} / p), P^{j}$ and $Q_{i}$.)

Lemma 3.4. Let $\rho_{p}=0 \in K_{*}^{M}(k) / p \cong H^{*}(\operatorname{Spec}(k) ; \mathbb{Z} / p)$. Then the Steenrod algebra $A_{p}$ acts on the $\bmod p$ etale cohomolgy $H_{\text {et }}^{*}(X ; \mathbb{Z} / p)$ compatible with that on the motivic cohomology.

Proof. In $H^{*, *^{\prime}}(\operatorname{Spec}(k) ; \mathbb{Z} / p)$, from Lemma 9.9 in $\mathrm{Vo} 4$, we know

$$
P^{i}(\tau)=0 \text { for } i>0 \text { and } \beta(\tau)=\rho_{p}=0 .
$$

(When $p=2$, this means $S q^{2 i}(\tau)=0, S q^{1}(\tau)=0$.)

When $p \geq 3$, the Cartan formula holds in the motivic cohomology (Proposition 9.7 in $\mathrm{Vo}$ ] $)$, and we have

$$
P^{i}(\tau x)=\tau P^{i}(x) \text { for } i>0 \text { and } \quad \beta(\tau x)=\tau \beta(x) .
$$

From the $B L(n, p)$ condition, $H_{e t}^{*}(X ; \mathbb{Z} / p)=\operatorname{colim}_{i} \tau^{i} H^{*, *^{\prime}}(X ; \mathbb{Z} / p)$, which implies the lemma.

For $p=2$, we also know from Proposition 9.7 in Vo4 that

$$
\begin{aligned}
S q^{2 *}(x y) & =\sum_{i} S q^{2 i}(x) S q^{2 *-2 i}(y)+\tau \sum_{i} S q^{2 i+1}(x) S q^{2 *-2 i-1}(y), \\
S q^{2 *+1}(x y) & =\sum_{j} S q^{j}(x) S q^{2 *+1-j}(y)+\rho_{2} \sum_{i} S q^{2 i+1}(x) S q^{2 *-2 i-1}(y) .
\end{aligned}
$$

Since $\rho_{2}=0$, we see $S q^{2 i+1}(\tau)=0$, and so $S q^{*}(\tau x)=\tau S q^{*}(x)$. This also induces the lemma.

Lemma 3.5. Let $\rho_{p}=0$. Then for each element $x$ in $H_{e t}^{*}(B G ; \mathbb{Z} / p)$, the images of cohomology (Bockstein, reduced) operations $\beta(x), P^{i}(x)$ for $i>0$ are in $N g(G)$ (hence $\left.x^{p} \in N g(G)\right)$. The image of the Gysin map $g_{*}(x)$ are also in $N g(G)$.

Proof. Let $x \in H^{*, *}(B G ; \mathbb{Z} / p)$. Then

$$
P^{i}(x) \in H^{*+2(p-1) i, *+(p-1) i}(B G ; \mathbb{Z} / p) .
$$

On the other hand, the corresponding etale cohomology operation $P_{e t}^{i}(x) \in$ $H_{e t}^{*}(X ; \mathbb{Z} / p)$ is identified as a cohomology operation on $H^{*, *}(B G ; \mathbb{Z} / p)$. So we have

$$
P_{e t}^{i}(x)=\tau^{(p-1) i} P^{i}(x) .
$$

Hence these elements are in $\operatorname{Im}(\tau)=N g(G)$. Similar facts hold for the Bockstein operation since $\beta_{\text {et }}(x)=\tau \beta(x)$.

For a smooth embedding $X \subset Y$ of codimension $c$, the Gysin map is defined on

$$
g_{*}: H^{*, *}(X ; \mathbb{Z} / p) \rightarrow H^{*+2 c, *+c}(Y ; \mathbb{Z} / p) .
$$

By the same reason as the cases of cohomology operations, we obtain the lemma. 
Here we give a sufficient condition for $x \notin N g(G)$. Recall the weight $w(x)=$ $2 *^{\prime}-*$ for the element (or operation) $x \in H^{*, *^{\prime}}(X ; \mathbb{Z} / p)$, e.g., $w(\tau)=2, w\left(Q_{i}\right)=-1$ and $w\left(P^{i}\right)=0$.

Lemma 3.6. Let $\rho_{p}=0$. For $x \in H_{\text {et }}^{n}(B G ; \mathbb{Z} / p)$, if $Q_{n-2} \ldots Q_{0}(x) \neq 0$, then $x \notin N g(G)$.

Proof. At first we consider the motivic cohomology. Identify $x$ as the element in $H^{n, n}(B G ; \mathbb{Z} / p)$. Suppose that $x=\tau \tilde{x}$. So $w(\tilde{x})=n-2$ since $w(\tau)=2$. The operation $Q_{i}$ decreases the weight by one. Let $\psi=Q_{n-2} \ldots Q_{0}(\tilde{x})$. Then $w(\psi)=-1$, so $\psi=0$.

On the other hand, in etale cohomology,

$$
c l(\psi)=Q_{n-2} \ldots Q_{0}(x) \neq 0 \in H_{e t}^{*}(B G ; \mathbb{Z} / p)
$$

for $c l=\left(\times \tau^{*-*^{\prime}}: H^{*, *^{\prime}}(B G ; \mathbb{Z} / p) \rightarrow H_{e t}^{*}(B G ; \mathbb{Z} / p)\right)$. This is a contradiction.

\section{Cohomological invariant}

Recall that $H^{1}(k ; G)$ is the first non-abelian Galois cohomology set of $G$, which represents the set of $G$-torsors over $k$. It is very important to study $H^{1}(k ; G)$; for example, $H^{1}\left(k ; O_{n}\right)$ is isomorphic to the set of isomorphism classes of nondegenerate quadratic forms over $k$ of rank $n$. (For details, see the excellent book Ga-Me-Se.)

The cohomological invariant is defined by

$$
\operatorname{Inv}^{i}(G, \mathbb{Z} / p)=\operatorname{Func}\left(H^{1}(F ; G) \rightarrow H^{i}(F ; \mathbb{Z} / p)\right),
$$

where Func means the additive group of the morphisms of functors $H^{1}(F ; G)$ $\rightarrow H^{i}(F ; \mathbb{Z} / p)$ on the category of field extensions $F / k$. (For the details, see Ga-Me-Se.) The cohomological invariant is studied by many authors. The cohomological invariants $\operatorname{Inv}^{*}(G ; \mathbb{Z} / p)$ are computed (for example in Ga-Me-Se] for elementary abelian 2-groups, $\mathrm{O}_{n}, S \mathrm{O}_{n}, \mathrm{G}_{2}, \ldots$ It is also stated in Ga-Me-Se that for many $G$ (but not all) $\operatorname{Inv}^{*}(G ; \mathbb{Z} / p)$ are detected by $\operatorname{Inv} v^{*}(H ; \mathbb{Z} / p)$ for elementary abelian $p$-subgroups $H$.

Recall $H^{*}\left(B G ; H_{\mathbb{Z} / p}^{*^{\prime}}\right)=\lim _{n \rightarrow \infty} H^{*}\left(U_{n} / G ; H_{\mathbb{Z} / p}^{*^{\prime}}\right)$ in the notation in $\S 3$. Let $x \in$ $H^{0}\left(B G ; H_{\mathbb{Z} / p}^{i}\right)$. Given a $G$-torsor $E$ over $F$, we can construct $x(E) \in H_{e t}^{i}(F ; \mathbb{Z} / p)$ as follows. Roughly speaking, we can identify $E$ as the pullback of the universal $G$-bundle by some map $f_{E}: \operatorname{Spec}(F) \rightarrow B G$. So we can take $x(E)=f_{E}^{*}(x) \in$ $H^{0}\left(\operatorname{Spec}(F) ; H_{\mathbb{Z} / p}^{i}\right)=H_{\text {et }}^{i}(F ; \mathbb{Z} / p)$. Indeed, Totaro proved Ga-Me-Se the following theorem in a letter to Serre.

Theorem 4.1 (Totaro). $\operatorname{Inv}^{*}(G ; \mathbb{Z} / p) \cong H^{0}\left(B G ; H_{\mathbb{Z} / p}^{*}\right)$.

Therefore we see

Corollary 4.2. $\operatorname{Im}\left(\psi_{\text {et }}^{*}\right) \cong H^{*}(B G ; \mathbb{Z} / p) /(N g) \subset \operatorname{Inv}(G ; \mathbb{Z} / p)$.

\section{ABELian $p$-GRoups}

In $\S 5-\S 8$, we will study $H^{*}(B G ; \mathbb{Z} / p) /(N g)$ for all concrete groups $G$, of which $\operatorname{Inv}^{*}(G ; \mathbb{Z} / p)$ are treated (and computed) in the book Ga-Me-Se. In this section we will first consider the case where $G$ is an abelian $p$-group.

Let us write $H^{*, *^{\prime}}=H^{*, *^{\prime}}(\operatorname{Spec}(k) ; \mathbb{Z} / p)$ and $H^{*}=H^{*, *}=K_{*}^{M}(k) / p$ so that $H^{*, *^{\prime}} \cong H^{*}[\tau]$. Moreover, let us simply write $H^{*}(B G ; \mathbb{Z} / p)=H_{e t}^{*}(B G ; \mathbb{Z} / p)$. 
First consider the case $G=\mathbb{Z} / p^{r}$. We assume that $k$ has a primitive $p^{r}$-th root of unity. The motivic cohomology of $B \mathbb{G}_{m}=\mathbb{P}^{\infty}$ is given as

$$
H^{*, *^{\prime}}\left(\mathbb{P}^{\infty} ; \mathbb{Z} / p\right) \cong H^{*, *^{\prime}}[y] \text { with } \operatorname{deg}(y)=(2,1) .
$$

In the stable $\mathbb{A}^{1}$-homotopy category, we have the cofibering (see (6.2) in $\mathrm{Vo}$ ] for details)

$$
B \mathbb{Z} / p^{r} \rightarrow E \stackrel{q_{r}}{\rightarrow} T h(E),
$$

where $E=O\left(-p^{r}\right)$ is the $-p^{r}$-th twisted line bundle (of the canonical one) of $\mathbb{P}^{\infty}$, $T h(E)$ is its Thom space and $q_{r}$ is the composition map with $p^{r}$-times twist on $\mathbb{P}^{\infty}$ and quotient map. Hence we have the long exact sequence

$$
\rightarrow H^{*-2, *^{\prime}-1}\left(\mathbb{P}^{\infty} ; \mathbb{Z} / p\right) \stackrel{q_{r}^{*}}{\rightarrow} H^{*, *^{\prime}}\left(\mathbb{P}^{\infty} ; \mathbb{Z} / p\right) \rightarrow H^{*, *^{\prime}}\left(B \mathbb{Z} / p^{r} ; \mathbb{Z} / p\right) \rightarrow .
$$

Here we see $q_{r}^{*}(x)=p^{r} y x=0$ for all $x \in H^{*, *^{\prime}}\left(\mathbb{P}^{\infty} ; \mathbb{Z} / p\right)$. Hence we can compute (as Voevodsky computed in [Vo1, Vo4 for $B \mathbb{Z} / p$ )

$$
H^{*, *^{\prime}}\left(B \mathbb{Z} / p^{r} ; \mathbb{Z} / p\right) \cong H^{*, *^{\prime}}[y(r)] \otimes\{1, x(r)\},
$$

where $\operatorname{deg}(y(r))=(2,1), \operatorname{deg}(x(r))=(1,1)$ for all primes $p$.

Remark. If we take $\mu_{p^{r}}$ instead of $\mathbb{Z} / p^{r}$, then the above fact holds without the existence of the $p^{r}$-th root of the unity. However, we only consider $\mathbb{Z} / p^{r}$ in this paper for the ease of arguments.

When $p=2$ and $r=1$, we know by Voevodsky Vo2, Vo4 that

$$
x(1)^{2}=\tau y(1)+\rho_{2} x(1) \quad \text { with } \rho_{2}=(-1) \in H^{1}=k^{*} /\left(k^{*}\right)^{2} .
$$

For the inclusion $i: \mathbb{Z} / p^{s} \subset \mathbb{Z} / p^{r}$ and quotient map $q: \mathbb{Z} / p^{r} \rightarrow \mathbb{Z} / p^{s}$, for $r>s$, we have

$$
i^{*}(y(r))=y(s), i^{*}(x(r))=0, \quad q^{*}(y(s))=0, q^{*}(x(s))=x(r) .
$$

Considering the map $q^{*}$ for $s=1$, we see $x(r)^{2}=0$ when $r>1$ and $p=2$ (note that $\rho_{2}=0$ by the assumption of the existence of the $2^{r}$-th root of unity). Of course $x(r)^{2}=0$ for $p>2$ and all $r \geq 1$.

Moreover, we see $\operatorname{Ker}(\tau) \mid H^{*, *^{\prime}}\left(B \mathbb{Z} / p^{r} ; \mathbb{Z} / p\right)=0$. Hence

$$
\operatorname{Inv} v^{*}\left(\mathbb{Z} / p^{r} ; \mathbb{Z} / p\right) \cong H^{*, *}\left(B \mathbb{Z} / p^{r} ; \mathbb{Z} / p\right) /(\tau)=H^{*}\{1, x(r)\} .
$$

For each space $X$, considering the cofibering

$$
B \mathbb{Z} / p^{r} \times X \rightarrow E \times X \stackrel{q^{r} \times i d}{\rightarrow} T h(E) \times X
$$

(

$$
H^{*, *^{\prime}}\left(B \mathbb{Z} / p^{r} \times X ; \mathbb{Z} / p\right) \cong H^{*, *^{\prime}}\left(B \mathbb{Z} / p^{r} ; \mathbb{Z} / p\right) \otimes_{H^{*, *^{\prime}}} H^{*, *^{\prime}}(X ; \mathbb{Z} / p) ;
$$

namely, the Kunneth formula holds.

Let $G=\mathbb{Z} / p^{r_{1}} \oplus \cdots \oplus \mathbb{Z} / p^{r_{s}}$ and assume the existence of the $p^{r_{i}}$-th root of unity. The motivic cohomology is given by the Kunneth formula

$$
H^{*, *^{\prime}}(B G ; \mathbb{Z} / p) \cong H^{*, *^{\prime}}\left[y_{1}\left(r_{1}\right), \ldots, y_{s}\left(r_{s}\right)\right] \otimes \Lambda\left(x_{1}\left(r_{1}\right), \ldots, x_{s}\left(r_{s}\right)\right)
$$


with $\operatorname{deg}\left(y_{i}\left(r_{i}\right)\right)=(2,1)$ and $\operatorname{deg}\left(x_{i}\left(r_{i}\right)\right)=(1,1)$ (when $p=2$ and $r_{i}=1, x_{i}(1)^{2}=$ $\left.\tau y_{i}(1)+\rho x_{i}(1)\right)$.

Theorem 5.1. Let $G$ be an abelian p-group, i.e., $G=\bigoplus_{i} \mathbb{Z} /\left(p^{r_{i}}\right)$. Suppose that there are primitive $p^{r_{i}}$-th roots of unity. Then

$$
\operatorname{Inv}^{*}(G ; \mathbb{Z} / p) \cong H^{*}(G ; \mathbb{Z} / p) /(N g) \cong H^{*} \otimes \Lambda\left(x_{1}\left(r_{1}\right), \ldots, x_{s}\left(r_{s}\right)\right),
$$

where if $p=2$ and $r_{i}=1$, then $x_{i}(1)^{2}=\rho x_{i}(1)$.

The elementary 2-group cases are stated in Theorem 16.4 in [Ga-Me-Se]. For ease of notation, let us write $x_{i}=x_{i}(1)$ and $y_{i}=y_{i}(1)$. The $Q_{i}$-operation acts on $H^{*, *^{\prime}}(B \mathbb{Z} / p ; \mathbb{Z} / p)$ by $Q_{i}(x)=y^{p^{i}}$ (while $Q_{i}(x(j))=0$ for all $j>1$ ). We consider the $Q_{i}$ action on $H^{*, *^{\prime}}\left(B(\mathbb{Z} / p)^{s} ; \mathbb{Z} / p\right)$. Each $Q_{i}$ is a derivation with $\bmod \left(\rho_{2}\right)$ (when $p>2, Q_{i}$ is always a derivation), and hence

$$
Q_{0} \ldots Q_{s-1}\left(x_{1} \ldots x_{s}\right)=\sum \operatorname{sgn}\left(j_{1}, \ldots, j_{s}\right) y_{1}^{p^{j_{1}}} y_{2}^{p^{j_{2}}} \ldots y_{s}^{p^{j_{s}}} \neq 0 \bmod \left(\rho_{2}\right),
$$

where $\left(j_{1}, \ldots, j_{s}\right)$ are permutations of $(0, \ldots, s-1)$. Thus we see that this case satisfies the sufficient condition of Lemma 3.6; in fact, $x_{1} \ldots x_{s} \notin N g(G)$.

Let us say that an element $\bar{x} \in H^{*}(B G ; \mathbb{Z} / p) /(N g)$ is detected by an elementary abelian $p$-subgroup $A$ if $\operatorname{Res}(\bar{x}) \neq 0$ for

$$
\text { Res }: H^{*}(B G ; \mathbb{Z} / p) /(N g) \rightarrow H^{*}(B A ; \mathbb{Z} / p) /(N g) .
$$

Lemma 5.2. Let $k$ be an algebraically closed field and $x \in H^{n}(B G ; \mathbb{Z} / p)$. If the corresponding element $\bar{x} \in H^{n}(B G ; \mathbb{Z} / p) /(N g)$ is detected by an elementary abelian p-subgroup, then $Q_{n-1} \ldots Q_{0}(x) \neq 0$ in $H^{*}(B G ; \mathbb{Z} / p)$.

Proof. Let $A$ be an elementary $p$-subgroup of $G$ such that

$$
\operatorname{Res}(\bar{x}) \neq 0 \in H^{*}(B A ; \mathbb{Z} / p) /(N g) \cong \Lambda\left(x_{1}, \ldots, x_{N}\right) .
$$

Take a subgroup $A^{\prime}$ of $A$ of $\operatorname{rank}_{p}\left(A^{\prime}\right)=n$ such that

$$
\operatorname{Res}(\bar{x})=x_{1} \ldots x_{n} \in H^{*}\left(B A^{\prime} ; \mathbb{Z} / p\right) /(N g) \cong \Lambda\left(x_{1}, \ldots, x_{n}\right) .
$$

Then we can take the restriction for motivic cohomology,

$$
\operatorname{Res}_{M O}(x)=x_{1} \ldots x_{n}+y \in H^{*, *^{\prime}}\left(B A^{\prime} ; \mathbb{Z} / p\right),
$$

where $y=\sum y_{I} x_{i_{1}} \ldots x_{i_{s}}$ for $s<n$ and $y_{I} \in \mathbb{Z} / p[\tau] \otimes \mathbb{Z} / p\left[y_{1}, \ldots, y_{n}\right]$. Here note that $Q_{0} \ldots Q_{n-1}(y)=0$, since $Q_{0} \ldots Q_{n-1}\left(x_{i_{1}} \ldots x_{i_{s}}\right)=0$. Thus we see in etale cohomology that

$$
\operatorname{Res}_{e t}\left(Q_{0} \ldots Q_{n-1} x\right)=Q_{0} \ldots Q_{n-1}\left(x_{1} \ldots x_{n}\right) \neq 0 \in H^{*}\left(B A^{\prime} ; \mathbb{Z} / p\right) .
$$

Hence $Q_{n-1} \ldots Q_{0}(x) \neq 0$ in $H^{*}(B G ; \mathbb{Z} / p)$.

\section{Cases $G=O_{n}$ AND $S O_{n}$}

Hereafter, in this paper we always assume that $k$ is an algebraically closed field in $\mathbb{C}$ unless otherwise stated.

lt is well known that

$$
\begin{aligned}
& H^{*}\left(B O_{n} ; \mathbb{Z} / 2\right) \cong H^{*}\left(\left(B(\mathbb{Z} / 2)^{n} ; \mathbb{Z} / p\right)^{S_{n}}\right. \\
& \cong \mathbb{Z} / 2\left[x_{1}, \ldots, x_{n}\right]^{S_{n}} \cong \mathbb{Z} / 2\left[w_{1}, \ldots, w_{n}\right],
\end{aligned}
$$


where $S_{n}$ is the $n$-th symmetric group and $w_{i}$ is the Stiefel-Whitney class representing the $i$-th elementary symmetric function of the $x_{i}$ 's. Let us write this by $y_{i}=x_{i}^{2}$. Then

$$
Q_{i-1} \ldots Q_{0}\left(w_{i}\right)=y_{1}^{p^{i-1}} y_{2}^{p^{i-2}} \ldots y_{i}+\ldots \neq 0 \in H^{*}\left(B(\mathbb{Z} / 2)^{n} ; \mathbb{Z} / 2\right),
$$

and hence $w_{i} \notin N g(G)$.

Recall the $\mathrm{Wu}$ formula

$$
S q^{i} w_{k}=\sum_{j=0}^{i}\left(\begin{array}{c}
k-j-1 \\
i-j
\end{array}\right) w_{k+i-j} w_{j} \quad(0 \leq i \leq k) .
$$

Many cases of products of $w_{i} w_{j}$ are in $N g(G)$, e.g., $w_{i}^{2} \in N g(G)$. More precisely, the motivic cohomology of $B O_{n}$ is computed for $k=\mathbb{C}$ (Theorem 8.1 in YYa2]):

$$
H^{*, *^{\prime}}\left(B O_{n} ; \mathbb{Z} / 2\right) \cong H^{*, *^{\prime}}\left(B(\mathbb{Z} / 2)^{n} ; \mathbb{Z} / 2\right)^{S_{n}} .
$$

In particular, we see $\operatorname{Ker}(\tau)=0$.

Given $x \in H^{*}(B G ; \mathbb{Z} / p)$, let us define the weight $w(x)$ as the smallest weight $w\left(x^{\prime}\right)$ such that $t_{\mathbb{C}}\left(x^{\prime}\right)=x$ with $x^{\prime} \in H^{*, *^{\prime}}(B G ; \mathbb{Z} / p)$. Note that monomials $\tau^{-j_{k}} w_{i_{1}} \ldots w_{i_{k}}$ do not make the homogeneous base of $H^{*, *^{\prime}}\left(B(\mathbb{Z} / 2)^{n} ; \mathbb{Z} / 2\right)^{S_{n}}$. For example,

$$
w\left(w_{2 i+1}+w_{2 i} w_{1}\right)=2 i-1 \quad \text { but } \quad w\left(w_{2 i+1}\right)=w\left(w_{2 i} w_{1}\right)=2 i+1 .
$$

This fact follows from $Q_{0}\left(w_{2 i}\right)=S q^{1}\left(w_{2 i}\right)=w_{2 i+1}+w_{2 i} w_{1}$.

In fact, the weight of the symmetric polynomial

$$
t=\sum x_{1}^{2 i_{1}+1} \ldots x_{k}^{2 i_{k}+1} x_{k+1}^{2 j_{1}} \ldots x_{k+q}^{2 j_{q}} \text { in } H^{*}\left((B \mathbb{Z} / 2)^{\times n} ; \mathbb{Z} / 2\right)
$$

(with $0 \leq i_{1} \leq \ldots \leq i_{k}, 0 \leq j_{1} \leq \ldots \leq j_{q}$ ) is given by $w(t)=k$.

Let $t \notin N g(G)$. Then there is $t^{\prime} \in H^{*, *}(B G ; \mathbb{Z} / p)$ (but not in $H^{*, *-1}(B G ; \mathbb{Z} / p)$ ) with $t_{\mathbb{C}}\left(t^{\prime}\right)=t$. So we have

$$
w(t)=w\left(t^{\prime}\right)=2 *-*=\operatorname{deg}(t) .
$$

This implies that $i_{1}=\ldots=j_{q}=0$, and hence $t$ is the symmetrization of $x_{1} \ldots x_{i}$, which is $w_{i}$.

Since $\operatorname{Ker}(\tau)=0$, we have the following theorem from Corollary 2.2.

\section{Theorem 6.1.}

$$
\operatorname{Inv}^{*}\left(O_{n} ; \mathbb{Z} / 2\right) \cong H^{*}\left(B O_{n} ; \mathbb{Z} / 2\right) /(N g) \cong \mathbb{Z} / 2\left\{1, w_{1}, \ldots, w_{n}\right\} .
$$

Indeed, $\operatorname{Inv}\left(O_{n} ; \mathbb{Z} / 2\right)$ is well known (Theorem 17.3 in Ga-Me-Se] for general $k$ :

$$
\operatorname{Inv}^{*}\left(O_{n} ; \mathbb{Z} / 2\right) \cong H^{*}\left\{1, w_{1}, \ldots, w_{n}\right\} .
$$

We consider the multiplicative structure of $\operatorname{Inv} v^{*}\left(O_{n} ; \mathbb{Z} / 2\right)$. We still know that $S q^{1}\left(w_{2 i}\right)=w_{2 i+1}+w_{2 i} w_{1} \in N g\left(O_{n}\right)$, and hence we have $w_{2 i+1}=w_{2 i} w_{1}$ in $\operatorname{Inv}^{*}\left(O_{n} ; \mathbb{Z} / 2\right)$.

By Rost and Kahn [Ka], the divided power operation can be defined in $K_{*}^{M}(F) / p$ compatible with fields $F$ over $k$ (and hence $\operatorname{Inv}^{*}(G ; \mathbb{Z} / p)$ ) if $\sqrt{ }-1 \in k$ (i. e. , $\rho=0$ in $\left.K_{1}^{M}(k) / 2\right)$. Vial Via] showed that the divided power operations are only compatible maps (natural maps) with field extensions over $k$. Moreover, Becher [Be] showed that $\gamma_{n}\left(w_{2}\right)=w_{2 n}$. (See also Milnor, p. 133 in [Mi].) 
Theorem 6.2 (Becher [Be, Via]). Let $\rho=0 \in K_{1}^{M}(k) / 2$. Then $\operatorname{Inv}^{*}\left(O_{n} ; \mathbb{Z} / 2\right)$ is generated by $w_{1}$ and $w_{2}$ as an $H^{*}$-ring with divided powers by

$$
\gamma_{i}\left(w_{2}\right)=w_{2 i}, \quad w_{2 i+1}=w_{2 i} w_{1} .
$$

Next consider the case $G=S O_{n}$. It is well known that

$$
H^{*}\left(B S O_{n} ; \mathbb{Z} / 2\right) \cong H^{*}\left(B O_{n} ; \mathbb{Z} / 2\right) /\left(w_{1}\right) \cong \mathbb{Z} / 2\left[w_{2}, \ldots, w_{n}\right] .
$$

Let $n=2 m+1=$ odd. For this case, there is the isomorphism

$$
O_{2 m+1} \cong S O_{2 m+1} \times \mathbb{Z} / 2 \text {. }
$$

Let $p: O_{n} \rightarrow S O_{n}$ be the projection and $i: S O_{n} \rightarrow O_{n}$ the inclusion. We consider the induced map $p^{*}$ and $i^{*}$ on the $\bmod 2$ motivic cohomology of their classifying spaces. Since $i^{*}\left(w_{1}\right)=0 \in H^{*, *^{\prime}}\left(B S O_{n} ; \mathbb{Z} / 2\right)$, we see that $w_{2 i+1} \in N g(G)$ from the above theorem (in fact, $S q^{1}\left(w_{2 i}\right)=w_{2 i+1}$ in $H^{*}\left(B S O_{n} ; \mathbb{Z} / 2\right)$ ). Moreover, $p^{*} w_{2 i}=w_{2 i} \neq 0 \bmod (N g(G))$ in $H^{*}\left(B O_{n} ; \mathbb{Z} / 2\right)$. We also note that $\operatorname{Ker}(\tau)=0$ for this case. Thus we have

Theorem 6.3. For $G=S O_{2 m+1}$, we have

$$
\operatorname{Inv}^{*}(G ; \mathbb{Z} / 2) \cong H^{*}(B G ; \mathbb{Z} / 2) /(N g) \cong \mathbb{Z} / 2\left\{1, w_{2}, \ldots, w_{2 m}\right\} .
$$

Moreover, $\operatorname{Inv}^{*}\left(S_{2 m+1} ; \mathbb{Z} / 2\right)$ is still computed (Theorem 19.1 in Ga-Me-Se] for general $k ; \operatorname{Inv}^{*}\left(S O_{2 m+1} ; \mathbb{Z} / 2\right) \cong H^{*}\left\{1, w_{2}, \ldots, w_{2 m}\right\}$.

Now consider the case $n=2 m=e v e n$. In this case the $\bmod 2$ motivic cohomology is not yet computed even $k=\mathbb{C}$ for $n>4$. However, we compute $H^{*, *}\left(B S O_{n} ; \mathbb{Z} / 2\right) /(\tau)$ easily. Consider the inclusion

$$
\mathrm{SO}_{2 m-1} \stackrel{i_{1}}{\rightarrow} \mathrm{SO}_{2 m} \stackrel{i_{2}}{\rightarrow} \mathrm{SO}_{2 m+1} .
$$

Since the restriction map for $i<m$,

$$
i_{1}^{*}\left(w_{2 i}\right) \notin N g\left(S O_{2 m-1}\right) \quad \text { and } \quad i_{2}^{*}\left(w_{2 i+1}\right) \in N g\left(S O_{2 m}\right),
$$

we see that $w_{2 i} \notin N g\left(S O_{2 m}\right)$ but $w_{2 i+1} \in N g\left(S O_{2 m}\right)$. Moreover, we know that $w\left(w_{2 m}\right)=2 m-2$ from Lemma 9.2 in [Ya2. In fact,

$$
w\left(w_{2 i}\right)=2 i \text { for } i<m \text { but } w\left(w_{2 m}\right)=2 m-2 .
$$

On the other hand, the monomial $w_{I}$ not of the form $w_{2 i}$ are all in $N g\left(S O_{2 m+1}\right)$, and so $i_{2}^{*}\left(w_{I}\right) \in N g\left(S O_{2 m}\right)$.

Thus we have

Theorem 6.4. $H^{*}\left(B S O_{2 m} ; \mathbb{Z} / 2\right) /(N g) \cong \mathbb{Z} / 2\left\{1, w_{2}, \ldots, w_{2 m-2}\right\}$.

Next we study $\operatorname{Inv}^{*}\left(S O_{2 m} ; \mathbb{Z} / 2\right)$. (Let $n=2 m$ as well.) There is an element (Lemma 9.3 in [Ya2]) in the motivic cohomology

$$
u_{m-1} \in H^{n, n-2}\left(B S O_{n} ; \mathbb{Z} / 2\right) \quad \text { with } \tau u_{m-1}=0 .
$$

So from Corollary 2.2, there is a non-zero element

$$
u \in H^{0}\left(B S O_{n} ; H_{\mathbb{Z} / 2}^{n-1}\right) /\left(H^{n-1, n-1}\left(B S O_{n} ; \mathbb{Z} / 2\right) /(\tau)\right) .
$$

Hence we can take $0 \neq u \in \operatorname{Inv}^{n-1}\left(S O_{n} ; \mathbb{Z} / 2\right)$, but it is only determined with $\bmod \left(H^{*}\left(B S O_{n} ; \mathbb{Z} / 2\right) /(N g)\right)$.

On the other hand, in Ga-Me-Se] it is proved (Theorem 20.6) for general $k$ :

$$
\operatorname{Inv}^{*}\left(S O_{2 m} ; \mathbb{Z} / 2\right) \cong H^{*}\left\{1, w_{2}, \ldots, w_{n-2}\right\} \oplus\left(\operatorname{Im}\left(I_{\delta}\right)\right) .
$$


Here, when $k=\mathbb{C}, \operatorname{Im}\left(I_{\delta}\right) \cong \mathbb{Z} / 2\{u\}$ with $\operatorname{deg}(u)=n-1$ from Proposition 20.1 in Ga-Me-Se. (Here $I_{\delta}$ can be taken canonically, but $u$ cannot.) Thus we see

Theorem 6.5. Let $G=S O_{2 m}$ and $m \geq 2$. Then for $\operatorname{deg}(u)=2 m-1$,

$$
\operatorname{Inv}^{*}(G ; \mathbb{Z} / 2) \cong H^{*}(B G ; \mathbb{Z} / 2) /(N g) \oplus \mathbb{Z} / 2\{u\} .
$$

From 22.10 in Ga-Me-Se, it is known that

$$
\text { Res }: \operatorname{Inv}^{*}(G ; \mathbb{Z} / p) \rightarrow \operatorname{Inv}^{*}(H ; \mathbb{Z} / p)
$$

is injective for $p=2, G=S O_{n}$ and $H=(\mathbb{Z} / 2)^{n-1}$. Hence for

$$
\operatorname{Res}_{H}: H^{0}\left(B S O_{n} ; H_{\mathbb{Z} / 2}^{*}\right) \rightarrow H^{0}\left(B(\mathbb{Z} / 2)^{n-1} ; H_{\mathbb{Z} / 2}^{*}\right)
$$

we have $\operatorname{Res}_{H}(u)=x_{1} \ldots x_{n-1}$. Of course $u \notin H^{*, *}\left(B S O_{n} ; \mathbb{Z} / 2\right) /(\tau)$, but $x_{1} \ldots x_{n-1} \in H^{*, *}\left(B(\mathbb{Z} / 2)^{n-1} ; \mathbb{Z} / 2\right) /(\tau)$. So note that for a map $X \rightarrow Y$, we see that

$$
f_{K}: \operatorname{Ker}(\tau)\left|H^{*+1, *^{\prime}-1}(Y ; \mathbb{Z} / p) \rightarrow \operatorname{Ker}(\tau)\right| H^{*+1, *^{\prime}-1}(X \mathbb{Z} / p)
$$

is (in general) not injective, even if $f_{H}: H^{*-*^{\prime}}\left(Y ; H_{\mathbb{Z} / p}^{*^{\prime}}\right) \rightarrow H^{*-*^{\prime}}\left(X ; H_{\mathbb{Z} / p}^{*^{\prime}}\right)$ is injective.

\section{7. $\operatorname{Spin}_{n}$ AND EXCEPTIONAL GROUPS}

By the definition, we have the short exact sequence

$$
0 \rightarrow \mathbb{Z} / 2 \rightarrow \operatorname{Spin}_{n} \stackrel{j}{\rightarrow} S O_{n} \rightarrow 1 .
$$

Recall that $w_{i}$ is the Stiefel-Whitney class in $H^{*}\left(B S O_{n} ; \mathbb{Z} / 2\right)$. Let $\Delta$ be a spin representation (which is any (orthogonal) representation which restricts to non-zero on $\mathbb{Z} / 2$ ). Let $w_{i}(\Delta)=\Delta^{*}\left(w_{i}\right)$ be the induced Stiefel-Whitney class. We also write by the same letter $w_{i}$ the Stiefel-Whitney class $j^{*}\left(w_{i}\right)$ of the usual representation $j: \operatorname{Spin}_{n} \rightarrow S O_{n}$.

Let $2^{h}$ be the the Radon-Hurwitz number (for details see $\mathrm{Qu}$, p. 210). Then the $\bmod (2)$ cohomology of $B \operatorname{Spin}_{n}$ was computed by Quillen:

$$
H^{*}\left(\text { BSpin }_{n} ; \mathbb{Z} / 2\right) \cong \mathbb{Z} / 2\left[w_{2^{h}}(\Delta)\right] \otimes \mathbb{Z} / 2\left[w_{2}, \ldots, w_{n}\right] /\left(w_{2}, Q_{i} w_{2} \mid 0 \leq i \leq h\right) .
$$

By the result of Becher (Theorem 6.2), we have

Theorem 7.1. $H^{*}\left(\operatorname{BSpin}_{n} ; \mathbb{Z} / 2\right) /(N g)=\mathbb{Z} / 2$ for $n>2$.

Proof. Recall representations $j: \operatorname{Spin}_{n} \rightarrow S O_{n}$ and $\Delta: \operatorname{Spin}_{n} \rightarrow S O_{N}$. We consider the induced map in Galois cohomology,

$$
j^{*}: H_{e t}^{*}\left(k\left(W_{*}\right)^{S O_{n}} ; \mathbb{Z} / 2\right) \rightarrow H_{e t}^{*}\left(k\left(W_{*}\right)^{\operatorname{Spin}_{n}} ; \mathbb{Z} / 2\right) .
$$

Moreover, by Rost and Kahn $\mathrm{Ka}$, the divided powers naturally act on $K_{*}^{M}(F) / p$ for a field $F$ over $k$.

Our case is $k=\mathbb{C}$. By Quillen's result, we see $j^{*}\left(w_{2}\right)=0$. From Becher's theorem (Theorem 6.2), we get

$$
j^{*}\left(w_{2 i}\right)=j^{*}\left(\gamma_{i}\left(w_{2}\right)\right)=\gamma_{i}\left(j^{*}\left(w_{2}\right)\right)=0 .
$$

It is known (Table (6.2) in Qu ) that $h>1$ for $n>2$ and hence $H^{2}\left(\right.$ BSpin $\left._{n} ; \mathbb{Z} / 2\right)$ $=0$. The fact that $w_{2}(\Delta)=0$ implies $w_{2^{h}}(\Delta)=0$ if $n>2$.

Corollary 7.2. For $n>2$ and $*>0$, there is the isomorphism

$$
\operatorname{Inv}^{*}\left(\operatorname{Spin}_{n} ; \mathbb{Z} / 2\right) \cong \operatorname{Ker}(\tau) \mid H^{*+1, *-1}\left(\operatorname{BSpin}_{n} ; \mathbb{Z} / 2\right) \text {. }
$$


The $\bmod (2)$ motivic cohomology of $B \operatorname{Spin}_{7}$ is computed in Theorem 9.6 in [Ya2, while it is determined only with the assumption of the existence of the Gottlieb transfer of motivic theories. We can easily see the above theorem also from concrete computation. Moreover, there are $\tau$-torsion elements

$$
y_{2} \in H^{4,2}\left(\operatorname{Spin}_{7} ; \mathbb{Z} / 2\right), \quad y_{2}^{\prime} \in H^{5,3}\left(\operatorname{BSpin}_{7} ; \mathbb{Z} / 2\right) .
$$

Therefore we can take $u \in H^{0}\left(\operatorname{BSpin}_{7}, H_{\mathbb{Z} / 2}^{3}\right), v \in H^{0}\left(\operatorname{BSpin}_{7}, H_{\mathbb{Z} / 2}^{4}\right)$.

Theorem 7.3 (Ga-Me-Se $]$ ).

$$
\operatorname{Inv}^{*}\left(\operatorname{BSpin}_{7} ; \mathbb{Z} / 2\right) \cong \mathbb{Z} / 2\{1, u, v\}, \quad|u|=3,|v|=4 .
$$

We consider exceptional Lie group types $G_{2}, F_{4}$ and split $E_{6}$. In Ga-Me-Se, it is proved (Theorem 18.1, Theorem 22.5) that

$$
\begin{gathered}
\operatorname{Inv}^{*}\left(G_{2} ; \mathbb{Z} / 2\right) \cong \operatorname{Inv}^{*}\left(E_{6} ; \mathbb{Z} / 2\right) \cong H^{*}\{1, u\}, \\
\operatorname{Inv}^{*}\left(F_{4} ; \mathbb{Z} / 2\right) \cong H^{*}\left\{1, u, f_{5}\right\} .
\end{gathered}
$$

(Unfortunately, we cannot re-explain $f_{5}$ by using $H^{*, *^{\prime}}\left(B F_{4} ; \mathbb{Z} / 2\right)$, since it has not yet been computed.)

Moreover, the restriction map of cohomological invariant for an elementary abelian 2-subgroup of rank 3 (resp. rank 5) is injective for $G_{2}, E_{6}$ (resp. for $\left.F_{4}\right)$; see 22.10 in Ga-Me-Se.

Theorem 7.4. Let $G=G_{2}, F_{4}, E_{6}$. Then $H^{*}(B G ; \mathbb{Z} / 2) /(N g)=\mathbb{Z} / 2$.

Proof. It is known by Borel (Bor, (6.8) in [Ko-Ya]), that the inclusion $i: G_{2} \rightarrow$ $\operatorname{Spin}_{7}$ induces the epimorphism $i^{*}: H^{*}\left(B \operatorname{Spin}_{7} ; \mathbb{Z} / 2\right) \rightarrow H^{*}\left(B G_{2} ; \mathbb{Z} / 2\right)$. Hence the result for $G=G_{2}$ follows from $H^{*}\left(\operatorname{BSpin}_{7} ; \mathbb{Z} / 2\right) /(N g)=\mathbb{Z} / 2$.

It is also known by Borel ( $\mathrm{Bor}$, page 786 in [Ko-Ya] $)$ that the inclusion $i^{\prime}$ : $\operatorname{Spin}_{9} \rightarrow F_{4}$ induces the injection $i^{\prime *}: H^{*}\left(B F_{4} ; \mathbb{Z} / 2\right) \rightarrow H^{*}\left(B \operatorname{Spin}_{9} ; \mathbb{Z} / 2\right)$. The groups $\operatorname{Spin}_{9}$ and $F_{4}$ have the same maximal abelian 2-group $H$ (of rank 5 ). We consider the restriction to this $H$. The fact that $\operatorname{Res}\left(H^{*}\left(\operatorname{BSpin}_{9} ; \mathbb{Z} / 2\right) /(N g)\right)$ $=\mathbb{Z} / 2$ implies the results for $F_{4}$, because the restriction is injective from Ga-Me-Se].

It is known that the inclusion $G_{2} \subset E_{6}$ induces the isomorphism

$$
\operatorname{Inv}^{*}\left(E_{6} ; \mathbb{Z} / 2\right) \cong \operatorname{Inv}^{*}\left(G_{2} ; \mathbb{Z} / 2\right)
$$

(page 52 in Ga-Me-Se]). Hence the result for $E_{6}$ follows from that for $G_{2}$.

Here we consider an example for the above theorem. The $\bmod (2)$ cohomology of $B G_{2}$ is well known:

$$
H^{*}\left(B G_{2} ; \mathbb{Z} / 2\right) \cong \mathbb{Z} / 2\left[w_{4}, w_{6}, w_{7}\right] .
$$

Let us write by $c_{4}, c_{6}, c_{7}$ the Chern classes with $c_{i}=w_{i}^{2}$.

Proposition 7.5 (鸟a2]). The cohomology $H^{*}\left(B G_{2} ; \mathbb{Z} / 2\right)$ is multiplicatively generated by $c_{4}, c_{6}, c_{7}$, and Image $\left(Q_{i}\right)$ for $0 \leq i \leq 2$, except for elements $w_{4}, w_{6}, w_{4} w_{6}$ and $w_{4} w_{6} w_{7}$.

The motivic cohomology $G_{2}$ is studied in Ya2. There we consider a virtual element $a$ with $\operatorname{deg}(a)=(3,3)$ so that $\tau^{7} c_{7} a=w_{6} w_{7} w_{4}, \tau Q_{0} a=w_{4}, \tau^{2} Q_{1} a=w_{6}$ and $\tau^{4} Q_{2} a=w_{6} w_{4}$. This virtual element $a$ exists in

$$
H^{0}\left(B G_{2} ; H_{\mathbb{Z} / 2}^{3}\right) \cong \operatorname{Inv}^{3}\left(G_{2} ; \mathbb{Z} / 2\right) \text {. }
$$


Indeed, this element restricts $x_{1} x_{2} x_{3}$ in $H^{0}\left(B(\mathbb{Z} / 2)^{3} ; H_{\mathbb{Z} / 2}^{3}\right.$ ) (as in the arguments before Theorem 7.4), and it represents the Rost cohomological invariant. So we see that $H^{*}\left(B G_{2} ; \mathbb{Z} / 2\right) /(N g) \cong \mathbb{Z} / 2$ as well. Moreover, we see there is

$$
y_{2} \in \operatorname{Ker}(\tau) \mid H^{4,2}\left(B G_{2} ; \mathbb{Z} / 2\right)
$$

as the case $\mathrm{SO}_{4}$.

Next we consider the odd prime cases where $(G, p)=\left(F_{4}, 3\right),\left(E_{6}, 3\right),\left(E_{7}, 3\right)$ or $\left(E_{8}, 5\right)$. In these cases, each exceptional Lie group has two conjugacy classes of maximal elementary abelian $p$-groups. One is the subgroup of a maximal torus and the other is a non-toral $A$. Let us write $i_{A}: A \rightarrow G$ and $i_{T}: T \rightarrow G$ as the inclusions. Tezuka and Kameko proved ( $\mathrm{Ka}$, Ka-Ya] $)$ that the following map is injective:

$$
i_{A}^{*} \times i_{T}^{*}: H^{*}(B G ; \mathbb{Z} / p) \rightarrow H^{*}(B A ; \mathbb{Z} / p) \times H^{*}(B T ; \mathbb{Z} / p) ;
$$

namely, $H^{*}(B G ; \mathbb{Z} / p)$ is detected by $A$ and $T$. Note that $H^{*}(B T ; \mathbb{Z} / p)$ is a polynomial algebra generated by 2 -dimensional elements of the weight degree zero, and so $H^{*}(B T ; \mathbb{Z} / p) /(N g) \cong \mathbb{Z} / p$.

On the other hand, in 22.10 in Ga-Me-Se, it is stated that the restriction map of $\operatorname{Inv}^{*}(G ; \mathbb{Z} / p)$ to some (maximal) elementary abelian $p$-group $H$ is an injection for these groups. Hence this is $H=A$ and

$$
H^{*}(B G ; \mathbb{Z} / p) /(N g) \subset H^{*}(B A ; \mathbb{Z} / p) /(N g) \cong \Lambda\left(x_{1}, \ldots, x_{s}\right) .
$$

Theorem 7.6. Let $(G, p)=\left(F_{4}, 3\right),\left(E_{6}, 3\right),\left(E_{7}, 3\right)$ or $\left(E_{8}, 5\right)$. Then

$$
H^{*}(B G ; \mathbb{Z} / p) /(N g)=\mathbb{Z} / p
$$

Proof. The restriction image of $H^{*}(B G ; \mathbb{Z} / p)$ to $A$ is studied in Ka-Ya. Images are generated as a ring by Chern classes and

$$
Q_{I}\left(x_{1} \ldots x_{s}\right) \in H^{*}(B A ; \mathbb{Z} / p) \cong \mathbb{Z} / p\left[y_{1}, \ldots, y_{s}\right] \otimes \Lambda\left(x_{1}, \ldots, x_{s}\right),
$$

where $Q_{I}=Q_{i_{1}} \ldots Q_{i_{r}}$ for some $I \neq \emptyset$. (Note that $x_{1} \ldots x_{s} \notin \operatorname{Im}\left(i_{A}^{*}\right)$.) Hence $i_{A}^{*}\left(H^{+}(B G ; \mathbb{Z} / p)\right) \in N g(A)$; that is, $\operatorname{Res}\left(H^{+}(B G ; \mathbb{Z} / p) /(N g)\right)=0$.

By the above injectivity results from [Ga-Me-Se, we see the desired result $H^{+}(B G ; \mathbb{Z} / p) /(N g)=0$.

Here we give an example of the above theorem. The $\bmod 3$ cohomology of $B F_{4}$ is completely determined by Toda.

Theorem 7.7 (Tod $)$. The cohomology $H^{*}\left(B F_{4} ; \mathbb{Z} / 3\right)$ is given by

$$
\mathbb{Z} / 3\left[z_{36}, z_{48}\right] \otimes\left(\mathbb{Z} / 3\left[z_{4}, z_{8}\right] \otimes\left\{1, z_{20}, z_{20}^{2}\right\}+\mathbb{Z} / 3\left[z_{26}\right] \otimes \Lambda\left(z_{9}\right) \otimes\left\{1, z_{20}, z_{21}, z_{25}\right\}\right)
$$

(here the above two terms have the intersection $\left\{1, z_{20}\right\}$ ), where subindices mean their degree, i. e. , $\left|z_{i}\right|=i$.

Indeed, we can compute ([Tod, [Ka-Ya] $) z_{26}\left|A=Q_{0} Q_{1} Q_{2}\left(u_{3}\right), z_{36}\right| A=c_{3,1}$, $z_{48}\left|A=c_{3,2}, z_{4}\right| A=Q_{0}\left(u_{3}\right), z_{8}\left|A=Q_{1}\left(u_{3}\right), z_{20}\right| A=Q_{2}\left(u_{3}\right), z_{9} \mid A=Q_{0} Q_{1}\left(u_{3}\right)$, $z_{21}\left|A=Q_{0} Q_{2}\left(u_{3}\right), z_{25}\right| A=Q_{1} Q_{2}\left(u_{3}\right)$. Here $u_{3}=x_{1} x_{2} x_{3}$ and $z_{36}$ and $z_{48}$ are represented by Chern classes of a minimal faithful complex representation. This shows that $H^{*}\left(B F_{4} ; \mathbb{Z} / 3\right) /(N g)=\mathbb{Z} / 3$ as well.

We have the Kunneth formula for the etale cohomology with coefficients $\mathbb{Z} / p$ (since $k$ is algebraically closed). Since $N g(G)$ is an ideal, we have the surjection

$$
H^{*}\left(B G_{1} ; \mathbb{Z} / p\right) /(N g) \otimes H^{*}\left(B G_{2} ; \mathbb{Z} / p\right) /(N g) \rightarrow H^{*}\left(B\left(G_{1} \times G_{2}\right) ; \mathbb{Z} / p\right) /(N g) .
$$


The above map is not an isomorphism, in general, because there is the possibility that $x_{1} \otimes x_{2} \in N g\left(G_{1} \times G_{2}\right)$ but $x_{1} \notin N g\left(G_{1}\right), x_{2} \notin N g\left(G_{2}\right)$.

However, when $H^{*}\left(B G_{i} ; \mathbb{Z} / p\right) /(N g)=\mathbb{Z} / p$, the above map is of course an isomorphism. If we can prove $H^{*}(B G ; \mathbb{Z} / p) /(N g)=\mathbb{Z} / p$ for $\left(E_{7}, p=2\right)$ and $\left(E_{8}, p=2,3\right)$, then the same fact holds for all 2-connected linear algebraic groups $G$.

\section{8. $P G L_{p}$}

Let $p$ be an odd prime and denote by $P G L_{p}$ the projective group which is the quotient of the general linear group $G L_{p}$ by the center $\mathbb{G}_{m}$. Its ordinary $\bmod (p)$ cohomology and the Chow ring are known by Vistoli $\mathrm{Vi}$ and Kameko-Yagita Ka-Ya].

To state the cohomology $H^{*}\left(B P G L_{p} ; \mathbb{Z} / p\right)$, we recall the Dickson algebra. Let $A \cong(\mathbb{Z} / p)^{n}$ be an elementary abelian $p$-group of rank $n$, and $H^{*}(B A ; \mathbb{Z} / p) \cong$ $\mathbb{Z} / p\left[y_{1}, \ldots, y_{n}\right] \otimes \Lambda\left(x_{1}, \ldots, x_{n}\right)$. The Dickson algebra is

$$
D_{n}=\mathbb{Z} / p\left[y_{1}, \ldots, y_{n}\right]^{G L_{n}\left(\mathbb{F}_{p}\right)} \cong \mathbb{Z} / p\left[c_{n, 0}, \ldots, c_{n, n-1}\right]
$$

with $\left|c_{n, i}\right|=2\left(p^{n}-p^{i}\right)$. The invariant ring under $S L_{n}\left(\mathbb{F}_{p}\right)$ is also given by

$$
S D_{n}=\mathbb{Z} / p\left[y_{1}, \ldots, y_{n}\right]^{S L_{n}\left(\mathbb{F}_{p}\right)} \cong D_{n}^{\prime} \otimes \mathbb{Z} / p\left[e_{n}\right],
$$

where $D_{n}=\mathbb{Z} / p\left[c_{n, 0}\right] \otimes D_{n}^{\prime}$, namely $D_{n}^{\prime}=\mathbb{Z} / p\left[c_{n, 1}, \ldots, c_{n, n-1}\right]$. Moreover, $e_{n}^{p-1}=$ $c_{n, 0}$. We also recall Mui's result by using $Q_{i}$ according to Kameko and Mimura Ka-Mi]:

$$
H^{*}(B A ; \mathbb{Z} / p)^{S L_{n}\left(\mathbb{F}_{p}\right)} \cong D_{n}^{\prime} \oplus S D_{n} \otimes \Lambda\left(Q_{0}, \ldots, Q_{n-1}\right)\left\{u_{n}\right\},
$$

where $u_{n}=x_{1} \ldots x_{n}$ and $e_{n}=Q_{0} \ldots Q_{n-1} u_{n}$.

Theorem 8.1 ( $\mathrm{Vi}$, [Ka-Ya $)$. There is an additive isomorphism

$$
H^{*}\left(B P G L_{p} ; \mathbb{Z} / p\right) \cong M \oplus N,
$$

where $M \cong \mathbb{Z} / p\left[z_{4}, z_{6}, \cdots, z_{2 p}\right]$ with $\operatorname{deg}\left(z_{i}\right)=i$ as modules (but not rings) and

$$
N \cong S D_{2} \otimes \Lambda\left(Q_{0}, Q_{1}\right)\left\{u_{2}\right\} \cong \mathbb{Z} / p\left[e_{2}, c_{2,1}\right] \otimes \Lambda\left(Q_{0}, Q_{1}\right)\left\{u_{2}\right\}
$$

with $\left|e_{2}\right|=2(p+1)$ and $\left|c_{2,1}\right|=2\left(p^{2}-p\right)$.

Here note that $e_{2}=Q_{0} Q_{1}\left(u_{2}\right)$, and $c_{2,1}$ is represented by some Chern class and $c_{2,1} \in M$ in the above theorem (but $e_{2}, c_{2,1} e_{2} \in N$ ).

Theorem 8.2 ( $\mathrm{Vi}$, Ka-Ya]). The cycle map

$$
c l: C H^{*}\left(B P G L_{p}\right) / p \rightarrow H^{2 *}\left(B P G L_{n} ; \mathbb{Z} / p\right)
$$

is injective and

$$
C H^{*}\left(B P G L_{p}\right) / p \cong \operatorname{Im}(c l)=M \oplus S D_{2}\left\{Q_{0} Q_{1} u_{2}\right\} .
$$

Hence we have from Lemmas 3.3 and 3.5

Theorem 8.3. $H^{*}\left(B P G L_{p} ; \mathbb{Z} / p\right) /(N g) \cong \mathbb{Z} / p\left\{1, u_{2}\right\}$ with $\left|u_{2}\right|=2$.

More strongly, it is shown in Ga-Me-Se that

$$
\operatorname{Inv}^{*}\left(P G L_{p} ; \mathbb{Z} / p\right) \cong H^{*}\left(B P G L_{p} ; \mathbb{Z} / p\right) /(N g) \cong \mathbb{Z} / p\left\{1, u_{2}\right\} .
$$


Throughout $\S 5-\S 8$, we have studied all groups $G$ which are studied in Ga-Me-Se] except for the symmetric group $S_{n}$. The permutations induce the natural representation $S_{n} \rightarrow O_{n}$. Let $A$ be the subgroup of $S_{n}$ generated by transpositions $(2 i-1,2 i)$ for $1 \leq i \leq[n / 2]$ so that $A \cong(\mathbb{Z} / 2)^{[n / 2]}$. Thus we have the map

$$
\operatorname{Inv}^{*}\left(O_{n} ; \mathbb{Z} / 2\right) \rightarrow \operatorname{Inv}^{*}\left(S_{n} ; \mathbb{Z} / 2\right) \rightarrow \operatorname{Inv}^{*}(A ; \mathbb{Z} / 2)^{S_{[n / 2]}} .
$$

It is shown in Ga-Me-Se] that the last map is isomorphic.

Theorem 8.4 ([Ga-Me-Se]). The cohomology $H^{*}\left(B S_{n} ; \mathbb{Z} / 2\right) /(N g)$ is isomorphic to

$$
\operatorname{Inv}^{*}\left(S_{n} ; \mathbb{Z} / 2\right) \cong \operatorname{Inv}^{*}\left(O_{[n / 2]} ; \mathbb{Z} / 2\right) \cong \mathbb{Z} / 2\left\{w_{1}, \ldots, w_{[n / 2]}\right\} .
$$

Moreover, it is shown in Ga-Me-Se] that $\operatorname{Inv}^{*}\left(S_{n} ; \mathbb{Z} / p\right)=\mathbb{Z} / p$ for $p \geq 3$. Unfortunately, we cannot find a good way to re-explain these facts from the cohomology $H^{*}\left(B S_{n} ; \mathbb{Z} / p\right)$, although they are quite well studied, e.g., Ad-Mi, Ma-Mi.

\section{Extraspecial $p$-Groups}

In $\S 11-\S 12$, we give examples of groups for undetected problems. The groups (special $p$-groups) are deeply related with extraspecial $p$ groups $p_{+}^{1+2 n}$. In this section we recall and explain the cohomology of extraspecial $p$-groups. Similar computations are used in $\S 11-\S 12$. In addition, we give some partial results for the cohomological invariant of $p_{+}^{1+2 n}$.

At first, we assume that $p$ is an odd prime. The extraspecial $p$-group $E_{n}=p_{+}^{1+2 n}$ (see $\S 4$ in Chapter 4 of $[\mathrm{Suz}]$ ) is the group such that its exponent is $p$, its center $Z(G)$ is $C \cong \mathbb{Z} / p$ and there is an extension

$$
0 \longrightarrow C \stackrel{i}{\longrightarrow} E_{n} \stackrel{\pi}{\longrightarrow} V \longrightarrow 0
$$

with $V=\bigoplus^{2 n} \mathbb{Z} / p$.

Remark. The definition of $2_{+}^{1+2 n}$ is quite different, e.g., exponent $\neq 2$; see the last parts of this section below. One of their reasons is the following. For each finite group $a, b, c \in G$, if $[a, b]=c \in Z(G)$, then we see (e.g., (3.7) in Chapter 4 of [Suz])

$$
a^{n} b^{n}=(a b)^{n} c^{n(n-1) / 2},
$$

e.g., if $a, b$ and $c$ are order $p$, then so is $a b$ for each odd $p$, but not for $p=2$.

We can take generators $a_{1}, \ldots, a_{2 n}, c \in E_{n}$ such that $\pi\left(a_{1}\right), . ., \pi\left(a_{2 n}\right)$ (resp. $c$ ) make a base of $V$ (resp. $C$ ) such that

$$
\left[a_{2 i-1}, a_{2 i}\right]=c \text { for } 1 \leq i \leq n
$$

and other pairs commute. We note that $E_{n}$ is also the central product of the $n$-copies of $E_{1}$ (Theorem 4.18 (i) in Chapter 4 of [Suz]):

$$
E_{n} \cong E_{1} \cdots E_{1}=E_{1} \times\langle c\rangle E_{1} \ldots \times\langle c\rangle E_{1} .
$$

Let us write the cohomologies (for details, see Te-Ya

$$
\begin{gathered}
H^{*}(B C ; \mathbb{Z} / p) \cong \mathbb{Z} / p[u] \otimes \Lambda(z), \quad \beta z=u, \\
H^{*}(B V ; \mathbb{Z} / p) \cong \mathbb{Z} / p\left[y_{1}, \ldots, y_{2 n}\right] \otimes \Lambda\left(x_{1}, \ldots, x_{2 n}\right), \quad \beta x_{i}=y_{i},
\end{gathered}
$$

identifying the dual of $a_{i}$ (resp. c) with $x_{i}$ (resp. $z$ ). These elements $x_{i}=\pi^{*}\left(x_{i}\right)$ also represent elements in $H^{*}\left(B E_{n} ; \mathbb{Z} / p\right)$ so that

$$
x_{i}: E_{n} \stackrel{p r}{\rightarrow}\left\langle a_{i}\right\rangle \cong \mathbb{Z} / p \text { in } \operatorname{Hom}\left(E_{n} ; \mathbb{Z} / p\right) \cong H^{1}\left(B E_{n} ; \mathbb{Z} / p\right) .
$$


The central extension is expressed by

$$
f=\sum_{i=1}^{n} x_{2 i-1} x_{2 i} \in H^{2}(B V ; \mathbb{Z} / p) .
$$

Hence $\pi^{*} f=0$ in $H^{2}\left(B E_{n} ; \mathbb{Z} / p\right)$. We consider the Hochshild-Serre spectral sequence

$$
E_{2}^{*, *^{\prime}} \cong H^{*}(B V ; \mathbb{Z} / p) \otimes H^{*^{\prime}}(B C ; \mathbb{Z} / p) \Longrightarrow H^{*}\left(B E_{n} ; \mathbb{Z} / p\right)
$$

Since $\pi^{*}(f)=0$, we see that $f=0$ in $E_{\infty}^{2,0}$. So there is an element $v \in E_{r}^{*,+}$ such that $d_{r}(v)=f$. By dimensional reason, it is the first non-zero differential $d_{2}(z)=f$.

The Cartan-Serre transgression theorem says (e.g., Theorem 4.8.1 in Ben]) that if $x \in E_{n}^{0, n-1}$ with $d_{n}(x)=y$, and $\phi$ is a Steenrod (reduced and Bockstein) operation of degree $r$, then $\phi(x)$ survives to $E_{n+r}^{0, n+r-1}$ and $d_{n+r}(\phi(x))=\phi(y)$.

Hence $u=Q_{0}(z)$ survives to $E_{3}^{0,2}$, which means that $d_{2}(u)=0$, and

$$
\begin{aligned}
& d_{3}(u)=d_{3}\left(Q_{0}(z)\right)=Q_{0}\left(d_{2}(z)\right)=Q_{0}(f)=Q_{0}\left(\sum x_{2 i-1} x_{2 i}\right) \\
& =\sum Q_{0}\left(x_{2 i-1}\right) x_{2 i}-x_{2 i-1} Q_{0}\left(x_{2 i}\right)=\sum y_{2 i-1} x_{2 i}-y_{2 i} x_{2 i-1} .
\end{aligned}
$$

Clearly, the above element is not in the ideal $(f)$. In particular,

$$
E_{4}^{*, 0} \cong \mathbb{Z} / p\left[y_{1}, \ldots, y_{2 n}\right] \otimes \Lambda\left(x_{1}, \ldots, x_{2 n}\right) /\left(f, Q_{0}(f)\right) \supset \Lambda\left(x_{1}, \ldots, x_{n}\right) /(f) .
$$

Lemma 9.1. The map $E_{3}^{*, 0} \rightarrow E_{\infty}^{*, 0} \subset H^{*}\left(B E_{n} ; \mathbb{Z} / p\right)$ induces the injection

$$
\Lambda\left(x_{1}, \ldots, x_{2 n}\right) /(f) \subset H^{*}\left(B E_{n} ; \mathbb{Z} / p\right) .
$$

Proof. We consider a similar group $E_{n}^{\prime}$ such that its center is $C \cong \mathbb{Z} / p$, and there is an extension

$$
0 \longrightarrow C \stackrel{i}{\longrightarrow} E_{n}^{\prime} \stackrel{\pi}{\longrightarrow} V^{\prime} \longrightarrow 0
$$

but $V^{\prime}=\bigoplus^{2 n} \mathbb{Z}_{p}$ such that there is the quotient map $q: E_{n}^{\prime} \rightarrow E_{n}$. We also consider the spectral sequence

$$
\bar{E}_{2}^{*, *^{\prime}} \cong H^{*}\left(B V^{\prime} ; \mathbb{Z} / p\right) \otimes H^{*}(B C ; \mathbb{Z} / p) \Longrightarrow H^{*}\left(B E_{n}^{\prime} ; \mathbb{Z} / p\right) .
$$

Here $H^{*}\left(B V^{\prime} ; \mathbb{Z} / p\right) \cong \Lambda\left(x_{1}, \ldots, x_{2 n}\right)$ since $y_{i}=0$ in $H^{*}\left(B V^{\prime} ; \mathbb{Z} / p\right)$. The first nonzero differential is $d_{2}(z)=f$. (Hence $d_{2}(u)=d_{2}\left(Q_{0}(z)\right)=0$ from the transgression theorem. ) So we have

$$
\bar{E}_{3}^{*, *^{\prime}} \cong \mathbb{Z} / p[u] \otimes\left(\Lambda\left(x_{1}, \ldots, x_{n}\right) /(f) \oplus K\right),
$$

where $K=\operatorname{Ker}\left(d_{2}\right) \mid \bar{E}_{2}^{*, 1} \subset \bar{E}_{2}^{*, *^{\prime}}$.

Here note that $\bar{E}_{3}^{*, *^{\prime}}$ is multiplicatively generated by elements $u$ and $\bar{E}_{3}^{*, *^{\prime}}$ with $*^{\prime} \leq 1$. However, we see that

$$
d_{3}(u)=\sum y_{2 i-1} x_{2 i}-y_{2 i} x_{2 i-1}=0
$$

Therefore all $d_{r}=0$ for all $r \geq 3$ by dimensional reason; that is, $\bar{E}_{3}^{*, *^{\prime}} \cong \bar{E}_{\infty}^{*, *^{\prime}}$. In particular, we have the injection

$$
\Lambda\left(x_{1}, \ldots, x_{n}\right) /(f) \subset \bar{E}_{3}^{*, 0} \cong \bar{E}_{\infty}^{*, 0} \subset H^{*}\left(B E_{n}^{\prime} ; \mathbb{Z} / p\right) .
$$


Hence from the map

$$
E_{3}^{*, 0} \rightarrow H^{*}\left(B E_{n} ; \mathbb{Z} / p\right) \rightarrow H^{*}\left(B E_{n}^{\prime} ; \mathbb{Z} / p\right),
$$

we obtain the result.

Unfortunately, we cannot yet prove

$$
\Lambda\left(x_{1}, \ldots, x_{2 n}\right) /(f) \subset H^{*}\left(B E_{n} ; \mathbb{Z} / p\right) /(N g) .
$$

However, when $n=1$, from Theorem 3.3 in Ya2 we see

Proposition 9.2. For an odd prime p, we have the isomorphism

$$
H^{*}\left(B p_{+}^{1+2} ; \mathbb{Z} / p\right) /(N g) \cong \mathbb{Z} / p\left\{1, x_{1}, x_{2}, a_{1}^{\prime}, a_{2}^{\prime}\right\} \quad \operatorname{deg}\left(a_{i}^{\prime}\right)=2 .
$$

When $p=2$, the situation becomes a bit better. The extraspecial 2-group $D(n)=2_{+}^{1+2 n}$ is defined (Theorem 4.18 in Chapter 4 of [Suz]) as the $n$-th central extension of the dihedral group $D_{8}$ of order 8. (Hence the exponent is 4.) It has the central extension

$$
0 \rightarrow \mathbb{Z} / 2 \rightarrow D(n) \rightarrow V \rightarrow 0
$$

with $V=\bigoplus^{2 n} \mathbb{Z} / 2$. Hence $H^{*}(B V ; \mathbb{Z} / 2) \cong \mathbb{Z} / 2\left[x_{1}, \ldots, x_{2 n}\right]$.

Then using the Hochschild-Serre spectral sequence, Quillen proved $\mathrm{Qu}$

$$
H^{*}(B D(n) ; \mathbb{Z} / 2) \cong \mathbb{Z} / 2\left[x_{1}, \ldots, x_{2 n}\right] /\left(f, Q_{0}(f), \ldots, Q_{n-2}(f)\right) \otimes \mathbb{Z}\left[w_{2^{n}}(\Delta)\right] .
$$

(In fact, when $n$ is the real case (i.e., $n=-1,0,1 \bmod (8))$, the cohomology $H^{*}\left(B_{S p i n} ; \mathbb{Z} / 2\right)$ injects into $H^{*}(B D(n) ; \mathbb{Z} / 2)$. $)$ Here $w_{2^{n}}(\Delta)$ is the Stiefel-Whitney class of any $2^{n}$-dimensional representation $\Delta$ which restricts non-zero on the center.

Moreover, Quillen proves the following two theorems (Theorem 5.10-11 in $\mathrm{Qu}$ ).

Theorem $9.3(\mathrm{Qu}) . H^{*}(B D(n) ; \mathbb{Z} / 2)$ is detected by the product of cohomoloogy of maximal elementary abelian subgroups.

Theorem 9.4 $(\mathrm{Qu})$. The non-zero Stiefel-Whitney $w_{i}(\Delta)$ are those of degrees $2^{n}$ and $2^{n}-2^{i}$ for $0 \leq i<n$.

In fact, $w_{i}(\Delta)$ generates the Dickson algebra in the cohomology of a maximal elementary abelian 2-group modulo the center of $D(n)$.

Proposition 9.5. When $n>2$, we have the surjection

$$
\Lambda\left(x_{1}, \ldots, x_{2 n}\right) /(f) \rightarrow H^{*}(B D(n) ; \mathbb{Z} / 2) /(N g) .
$$

Proof. The fact that $w_{2}(\Delta)=0$ follows from Quillen's second theorem above, since $n>2$. Hence we have $w_{2^{n}}(\Delta) \in N g(D(n))$ from Becher's theorem (Theorem 6.2). Of course $x_{i}^{2}=Q_{0}\left(x_{i}\right) \in N g(D(n))$. Thus we prove the theorem.

Considering the restriction to the subgroup $A=\left\langle x_{1}, x_{3}, \ldots, x_{2 n-1}\right\rangle$, we see that

$$
\Lambda\left(x_{1}, x_{3}, \ldots, x_{2 n-1}\right) \subset H^{*}(B D(n) ; \mathbb{Z} / 2) /(N g) .
$$

From Theorem $9.3, H^{*}(B D(n) ; \mathbb{Z} / 2)$ is detected by elementary abelian 2 -groups. However, we cannot yet see that this is so for $H^{*}(B D(n) ; \mathbb{Z} / 2) /(N g)$; namely, whether or not the above epimorphism is indeed an isomorphism. 


\section{NoETHER's PROBLEM}

In this section, we recall Noether's problem and consider the relation to the detected problem for $H^{*}(B G ; \mathbb{Z} / p) /(N g)$. In particular, we will show that for a $p$ group $G$ of exponent $p$, if $H^{2}(B G ; \mathbb{Z} / p) /(N g)$ is not detected by any $A \cong \mathbb{Z} / p \oplus \mathbb{Z} / p$, then $G$ is a counterexample of Noether's problem; namely, $k(W)^{G}$ is not purely transcendental over $k$.

Let $K$ be a finitely generated extension over $k$. We recall the definition of unramifed cohomology of $H^{*}(K ; \mathbb{Z} / p)$ according to Saltman, Peyre and ColliotThelene $[\mathrm{Sa}, \mathrm{Pe}, \mathrm{Co}$.

We denote by $P(K / k)$ the set of discrete valuation rings $A$ of rank one such that $k \subset A \subset K$ and that the fraction field $\operatorname{Fr}(A)$ of $A$ is $K$. If $A$ belongs to $P(K / k)$, then for the residue field $\kappa_{A}$, we can define the residue map $\partial_{A}: H^{*}(K ; \mathbb{Z} / p) \rightarrow$ $H^{*-1}\left(\kappa_{A} ; \mathbb{Z} / p\right)$ as follows.

Let $\hat{K}_{A}$ be the completion, $\hat{K}_{A}^{n r}$ the maximal unramified extension of $\hat{K}_{A}$ and $\bar{K}_{A}$ an algebraic closure of $\hat{K}_{A}$. Put $I_{A}=\operatorname{Gal}\left(\bar{K}_{A} / \hat{K}_{A}^{n r}\right)$ and $G_{A}=\operatorname{Gal}\left(\bar{K}_{A} / \hat{K}_{A}\right)$,

$$
\bar{K}_{A} \stackrel{I_{A}}{-} \hat{K}_{A}^{n r} \stackrel{G_{A} / I_{A}}{-} \hat{K}_{A}--K
$$

Then $\partial_{A}$ is defined as the composition of maps

$$
\begin{gathered}
\partial_{A}: H^{*}(K ; \mathbb{Z} / p) \rightarrow H^{*}\left(\hat{K}_{A} ; \mathbb{Z} / p\right) \\
\stackrel{\text { proj. }}{\rightarrow} H^{*-1}\left(G_{A} / I_{A} ; \mathbb{Z} / p\right) \otimes H^{1}\left(I_{A} ; \mathbb{Z} / p\right) \cong H^{*-1}\left(\kappa_{A} ; \mathbb{Z} / p\right) .
\end{gathered}
$$

Here we used the fact that $G \cong I_{A} \oplus\left(G_{A} / I_{A}\right)$ ([Sa] $)$ and $I_{A} \cong \hat{\mathbb{Z}}$. Moreover, $H^{*}(\hat{\mathbb{Z}} ; \mathbb{Z} / p) \cong \mathbb{Z} / p$ if $*=1$ and $\cong 0$ for $*>1$.

Then we can define the unramified cohomology

$$
H_{n r}^{*}(K ; \mathbb{Z} / p)=\bigcap_{A \in P(K / k)} \operatorname{Ker}\left(H^{*}(K ; \mathbb{Z} / p) \stackrel{\partial_{A}}{\rightarrow} H^{*-1}\left(\kappa_{A} ; \mathbb{Z} / p\right)\right) .
$$

Hereafter (to the end of this paper) we assume that $G$ is a finite group. According to Peyre $\underline{\mathrm{Pe}}$, we define a subring $H_{n r}^{*}(G ; \mathbb{Z} / p)$ of $H^{*}(B G ; \mathbb{Z} / p)$ as follows. Let $P(G)$ be the set of elements $g \in G$ such that $I=\langle g\rangle \cong \mathbb{Z} / p^{s}$ for some $s \geq 1$ but $g \neq h^{p}$ for any $h \in G$. Then the centralizer is written as $Z_{G}(I) \cong I \oplus H$ with $H=Z_{G}(I) / I$. Let us write by $\partial_{g}$ the composition map

$$
\begin{gathered}
\partial_{g}: H^{*}(B G ; \mathbb{Z} / p) \rightarrow H^{*}\left(B Z_{G}(I) ; \mathbb{Z} / p\right) \\
\stackrel{\text { proj. }}{\rightarrow} H^{*-1}(B H ; \mathbb{Z} / p) \otimes H^{1}(B I ; \mathbb{Z} / p) \cong H^{*-1}(B H ; \mathbb{Z} / p)
\end{gathered}
$$

using $H^{1}(B I ; \mathbb{Z} / p) \cong \mathbb{Z} / p$. Then define the unramified cohomology by

$$
H_{n r}^{*}(G ; \mathbb{Z} / p)=\bigcap_{g \in P(G)} \operatorname{Ker}\left(H^{*}(B G ; \mathbb{Z} / p) \stackrel{\partial_{q}}{\rightarrow} H^{*-1}(B H ; \mathbb{Z} / p)\right) .
$$

From the above two definitions of unramified cohomologies, we have the following theorem.

Theorem 10.1 (Peyre, $[\mathrm{Pe})$. Let $V$ be a faithful representation of $G$. Recall that $q$ is the quotient map $q: \operatorname{Gal}\left(\bar{k}(\bar{V}) / k(V)^{G}\right) \rightarrow G$. Then we have $q^{*}\left(H_{n r}^{*}(G ; \mathbb{Z} / p)\right) \subset$ $H_{n r}^{*}\left(k(V)^{G} ; \mathbb{Z} / p\right)$. 
Remark. We can define the similar map

$$
\bar{\partial}_{g}: H^{*}(B G ; \mathbb{Z} / p) /(N g) \rightarrow H^{*-1}(B H ; \mathbb{Z} / p) /(N g)
$$

using $H^{1}(B I ; \mathbb{Z} / p) \cong H^{1}(B I ; \mathbb{Z} / p) /(N g) \cong \mathbb{Z} / p$. We also define the unramified cohomology by

$$
\bar{H}_{n r}^{*}(G ; \mathbb{Z} / p)=\bigcap_{g \in P(G)} \operatorname{Ker}\left(\bar{\partial}_{g}\right) \subset H^{*}(B G ; \mathbb{Z} / p) /(N g) .
$$

Then we can prove $q^{*}\left(\bar{H}_{n r}^{*}(G ; \mathbb{Z} / p)\right) \subset H_{n r}^{*}\left(k\left(W_{*}\right)^{G} ; \mathbb{Z} / p\right)$.

It is well known (Theorem 4.1.5 in [Co]) that if $K$ is purely transcendental over $k$ (i.e., $K \cong k\left(x_{1}, \ldots, x_{n}\right)$ for indeterminate $\left.x_{i}\right)$, then

$$
H^{*}(k ; \mathbb{Z} / p) \cong H_{n r}^{*}(K ; \mathbb{Z} / p) \text {. }
$$

(We assume $k$ is an algebraically closed field, and so $H^{+}(k ; \mathbb{Z} / p)=0$.) Hence if $K$ is purely transcendental over $k$, then $H_{n r}^{+}(K ; \mathbb{Z} / p)=0$.

Corollary 10.2. Let $x \in H^{+}(B G ; \mathbb{Z} / p)$ such that $x \in H_{n r}^{*}(G ; \mathbb{Z} / p)$ but $x \notin N g(G)$. Then $q^{*}(x) \neq 0$ in $H_{n r}^{*}\left(k\left(W_{*}\right)^{G} ; \mathbb{Z} / p\right)$. Hence $k\left(W_{*}\right)^{G} / k$ is not purely transcendental.

Proof. From the preceding theorem, we have

$$
q^{*}(x) \in H_{n r}^{*}\left(k\left(W_{*}\right)^{G} ; \mathbb{Z} / p\right) \subset H^{*}\left(k\left(W_{*}\right)^{G} ; \mathbb{Z} / p\right) .
$$

From the explanation just before Lemma 3.1, we see

$$
\psi_{\text {et }}^{*}=q^{*}: H^{*}(B G ; \mathbb{Z} / p) /(N g) \subset H_{Z a r}^{0}\left(B G ; H_{\mathbb{Z} / p}^{*}\right) \subset H^{*}\left(k\left(W_{*}\right)^{G} ; \mathbb{Z} / p\right) .
$$

Hence we see $q^{*}(x) \neq 0$.

Corollary 10.3. Let $G$ be a p-group of exponent $p$. If $H^{2}(B G ; \mathbb{Z} / p) /(N g) \neq 0$ and it is not detected by $\mathbb{Z} / p \times \mathbb{Z} / p$, then $k\left(W_{2}\right)^{G}$ is not purely transcendental.

Proof. Suppose that $x \notin H_{n r}^{2}(G ; \mathbb{Z} / p)$. Then there is $g$ such that $\partial_{g}(x) \neq 0$ for

$$
\partial_{g}: H^{2}(B G ; \mathbb{Z} / p) \stackrel{p r_{1}}{\rightarrow} H^{1}(B I ; \mathbb{Z} / p) \otimes H^{1}(B H ; \mathbb{Z} / p) \stackrel{p r_{2}}{\rightarrow} H^{1}(B H ; \mathbb{Z} / p) .
$$

So $\operatorname{pr}_{1}(x)=x_{1} \otimes x_{2} \neq 0$, where $x_{1} \in H^{1}(B I ; \mathbb{Z} / p) \cong \mathbb{Z} / p$ and $x_{2} \in H^{1}(B H ; \mathbb{Z} / p)$. Identify $x_{2} \in H o m(H ; \mathbb{Z} / p) \cong H^{1}(B H ; \mathbb{Z} / p)$. Note the exponent of $H$ is $p$. Hence we can take the subgroup $\mathbb{Z} / p \cong J \subset H$ such that $x_{2} \mid J \neq 0$.

Then the corresponding element $\bar{x} \in H^{2}(B G ; \mathbb{Z} / p) /(N g)$ is detected by $I \oplus J$, because $\operatorname{Res}(\bar{x})=\bar{x}_{1} \otimes \bar{x}_{2}$ for

$$
H^{1}(B I ; \mathbb{Z} / p) \otimes H^{1}(B J ; \mathbb{Z} / p) \cong H^{2}(B(I \oplus J) ; \mathbb{Z} / p) /(N g) \cong \mathbb{Z} / p\left\{\bar{x}_{1} \otimes \bar{x}_{2}\right\} .
$$

Hence if $\bar{x} \neq 0$ is not detected by each $\mathbb{Z} / p \oplus \mathbb{Z} / p$, then $x \notin N g(G)$ and $x \in$ $H_{n r}^{2}(G ; \mathbb{Z} / p)$. Thus we have the desired result from Corollary 10.2 .

\section{Saltman's example}

In $\S 11$ and $\S 12$, we assume $p \geq 3$. Saltman [Sa] showed that $H_{n r}^{2}\left(k\left(W_{2}\right)^{G s} ; \mathbb{Q} / \mathbb{Z}\right)$ $\neq 0$ for some special $p$-group $G s$. We will give a short proof of the fact that

$$
H_{n r}^{2}\left(k\left(W_{2}\right)^{G s} ; \mathbb{Z} / p\right) \neq 0 .
$$


A $p$-group $G$ is called a special $p$-group if $Z(G)=[G, G] \cong(\mathbb{Z} / p)^{s}$ for $s \geq 1$. (When $s=1$, we call it an extraspecial $p$-group.) Let $G s$ be the special $p$-group of exponent $p$ defined by

$$
\begin{gathered}
0 \rightarrow\left\langle c, c_{3}, c_{4}\right\rangle \rightarrow G s \rightarrow\left\langle a_{1}, a_{2}, a_{3}, a_{4}\right\rangle \rightarrow 0 \\
\text { with } \quad\left[a_{1}, a_{2}\right]=\left[a_{3}, a_{4}\right]=c, \quad\left[a_{1}, a_{3}\right]=c_{3}, \quad\left[a_{1}, a_{4}\right]=c_{4},
\end{gathered}
$$

and the other pairs commute. Note that

$$
G s /\left\langle c_{3}, c_{4}\right\rangle \cong p_{+}^{1+4}, \quad\left\langle c_{3}, a_{1}, a_{3}\right\rangle \cong p_{+}^{1+2}, \quad\left\langle c_{4}, a_{1}, a_{4}\right\rangle \cong p_{+}^{1+2} .
$$

Of course such a group Gs exists because the set

$$
\left\{c^{i_{1}} c_{3}^{i_{2}} \ldots a_{4}^{i_{7}} \mid 0 \leq i_{s} \leq p-1,1 \leq s \leq 7\right\}
$$

forms the group by the multiplication given by the commutator and $c^{p}=c_{3}^{p}=\ldots=$ $a_{4}^{p}=1$. (For $p=2$, there does not exist a Gs with exponent 2. See the Remark in $\S 9$ and see [Suz] for properties of special groups.)

Let us write

$$
\begin{gathered}
a^{\Lambda}=a_{1}^{\lambda_{1}} \ldots a_{4}^{\lambda_{4}} \quad \text { for } \Lambda=\left(\lambda_{1}, \ldots, \lambda_{4}\right) \in(\mathbb{Z} / p)^{4}, \\
a^{M}=a_{1}^{\mu} \ldots a_{4}^{\mu_{4}} \quad \text { for } M=\left(\mu_{1}, \ldots, \mu_{4}\right) \in(\mathbb{Z} / p)^{4} .
\end{gathered}
$$

The commutator is given by the definition

$$
\begin{gathered}
{\left[a^{\Lambda}, a^{M}\right]=c^{d} c_{3}^{d_{3}} c_{4}^{d_{4}}, \quad \text { where } d=\lambda_{1} \mu_{2}-\lambda_{2} \mu_{1}+\lambda_{3} \mu_{4}-\lambda_{4} \mu_{3},} \\
d_{3}=\lambda_{1} \mu_{3}-\lambda_{3} \mu_{1}, \quad d_{4}=\lambda_{1} \mu_{4}-\lambda_{4} \mu_{1} .
\end{gathered}
$$

Lemma 11.1. Let $\langle\Lambda, M\rangle$ and $\left\langle\Lambda^{\prime}, M^{\prime}\right\rangle$ generate the same 2-dimensional vector space in $(\mathbb{Z} / p)^{4}$. Then $\left[a^{\Lambda}, a^{M}\right]=0$ if and only if $\left[a^{\Lambda^{\prime}}, a^{M^{\prime}}\right]=0$.

Proof. First note by the definition of $G s$ that

$$
Z(G s)=[G s, G s]=\left\langle c, c_{3}, c_{4}\right\rangle .
$$

For each $\Lambda, M$, we see $a^{\Lambda} a^{M}=a^{\Lambda+M} c(\Lambda, M)$ with $c(\Lambda, M) \in Z(G s)$. We also note that $\left[a^{\Lambda} c(0), a^{M}\right]=\left[a^{\Lambda}, a^{M}\right]$ for each $c(0) \in Z(G s)$.

Suppose $\left[a^{\Lambda}, a^{M}\right]=0$. Let $\Theta_{i}=\beta_{i} \Lambda+\gamma_{i} M$ with $\beta_{i}, \gamma_{i} \in \mathbb{Z} / p$ for $i=1,2$. Note that

$$
\left[a^{\beta_{1} \Lambda} a^{\gamma_{1} M}, a^{\Lambda}\right]=\left[\left(a^{\Lambda}\right)^{\beta_{1}} c(1)\left(a^{M}\right)^{\gamma_{1}} c(2), a^{\Lambda}\right]=\left[\left(a^{\Lambda}\right)^{\beta_{1}}\left(a^{M}\right)^{\gamma_{1}}, a^{\Lambda}\right]=0
$$

for some $c(1), c(2) \in Z(G s)$, since $\left[a^{\Lambda}, a^{M}\right]=0$. Similarly, we have

$$
0=\left[a^{\beta_{1} \Lambda} a^{\gamma_{1} M}, a^{\lambda_{2} \Lambda} a^{\gamma_{2} M}\right]=\left[a^{\Theta_{1}} c(3), a^{\Theta_{2}} c(4)\right]=\left[a^{\Theta_{1}}, a^{\Theta_{2}}\right]
$$

for some $c(3), c(4) \in Z(G s)$.

Recall that $x_{i} \in H^{1}(B G s ; \mathbb{Z} / p)$ is the dual of $a_{i}$ (and $\left.\beta\left(x_{i}\right)=y_{i}\right)$; namely,

$$
x_{i}: G s \stackrel{p r .}{\rightarrow}\left\langle a_{i}\right\rangle \cong \mathbb{Z} / p \in \operatorname{Hom}(G s ; \mathbb{Z} / p) .
$$

Hence $x_{i} \mid\left\langle a^{\Lambda}\right\rangle=\lambda_{i}$. Moreover, the above central extension (representing Gs) is written as an element in $H^{2}\left(B(\mathbb{Z} / p)^{4} ;(\mathbb{Z} / p)^{3}\right)$,

$$
\left(x_{1} x_{2}+x_{3} x_{4}\right)\{c\}+x_{1} x_{3}\left\{c_{3}\right\}+x_{1} x_{4}\left\{c_{4}\right\}
$$

identifying $H^{*}\left(B(\mathbb{Z} / p)^{4} ;(\mathbb{Z} / p)^{3}\right) \cong H^{*}\left(B(\mathbb{Z} / p)^{4} ; \mathbb{Z} / p\right)\left\{c, c_{3}, c_{4}\right\}$ (see also [Te-Ya] for details). 
Lemma 11.2. The 3 -dimensional cohomology $H^{3}(B G s ; \mathbb{Z} / p)$ contains the $\mathbb{Z} / p$ module

$$
A=\mathbb{Z} / p\left\{y_{i} x_{j} \mid 1 \leq i, j \leq 4\right\} /\left(Q_{0}(f), Q_{0}\left(x_{1} x_{3}\right), Q_{0}\left(x_{1} x_{4}\right)\right) .
$$

Proof. Consider the central extension

$$
0 \rightarrow\left\langle c, c_{3}, c_{4}\right\rangle \rightarrow G \rightarrow V=\left\langle a_{1}, \ldots, a_{4}\right\rangle \rightarrow 0
$$

and the induced spectral sequence

$$
E_{2}^{*, *^{\prime}} \cong \mathbb{Z} / p\left[y_{1}, \ldots, y_{4}, u, u_{3}, u_{4}\right] \otimes \Lambda\left(x_{1}, \ldots, x_{4}, z, z_{3}, z_{4}\right)
$$

converging to $H^{*}(B G s ; \mathbb{Z} / p)$.

The first non-zero differential is computed as the cases of extraspecial $p$-groups

$$
d_{2}(z)=f, \quad d_{2}\left(z_{3}\right)=x_{1} x_{3}, \quad d_{2}\left(z_{4}\right)=x_{1} x_{4} .
$$

The second non-zero differential is

$$
d_{3}(u)=Q_{0}(f), \quad d_{3}\left(u_{3}\right)=Q_{0}\left(x_{1} x_{3}\right), \quad d_{3}\left(u_{4}\right)=Q_{0}\left(x_{1} x_{4}\right) .
$$

Thus we see $A \cong E_{4}^{3,0} \cong E_{\infty}^{3,0} \subset H^{3}(B G ; \mathbb{Z} / p)$ by dimensional reasons of differentials.

Theorem 11.3 (Saltman, [Sa]). We have

$$
x_{1} x_{2} \in H_{n r}^{2}(G s ; \mathbb{Z} / p) \quad \text { but } \quad x_{1} x_{2} \notin N g(G s) .
$$

Hence $k\left(W_{2}\right)^{\text {Gs }}$ is not purely transcendental.

Proof. From the preceding lemma, we see that $Q_{0}\left(x_{1} x_{2}\right) \neq 0$ in $H^{3}(B G s ; \mathbb{Z} / p)$. Hence we have $x_{1} x_{2} \notin N g(G s)$ from Lemma 3.6.

Next we will show $x_{1} x_{2} \in H_{n r}(G s ; \mathbb{Z} / p)$. Suppose this is not the case. By the definition (or Corollary 10.3), this means that there are elements $g \in G s$ and $h \in Z_{G s}(\langle g\rangle)$ such that

$$
x_{i}\left|\langle g\rangle \neq 0, \quad x_{j}\right|\langle h\rangle \neq 0 \quad \text { for }\{i, j\}=\{1,2\} .
$$

Let $g=a^{\Lambda} c(g)$ with $c(g) \in Z(G s)$. From Lemma 11.1, we can take

$$
\lambda_{1}=1, \lambda_{2}=0, \quad \Lambda=\left(1,0, \lambda_{3}, \lambda_{4}\right) .
$$

(Hence $x_{1} \mid\langle g\rangle=1$ and $x_{2} \mid\langle g\rangle=0$. ) Then we can take $h=a^{M} \in Z(\langle g\rangle)$ so that $x_{2} \mid\langle h\rangle=1$ and $h \notin\langle g\rangle$, which means that

$$
\mu_{1}=0, \mu_{2}=1, \quad M=\left(0,1, \mu_{3}, \mu_{4}\right) .
$$

Since $[h, g]=0$, we see that $d_{3}=0$ and $d_{4}=0$. Hence we have

$$
\mu_{3}=\lambda_{3} \mu_{1}=0 \quad \text { and } \quad \mu_{4}=\lambda_{4} \mu_{1}=0
$$

(and hence $M=(0,1,0,0))$. Therefore $d=1 \times 1-0+0-0=1$. This is a contradiction. Hence we have proved $x_{1} x_{2} \in H_{n r}(G s ; \mathbb{Z} / p)$. 


\section{NON-DETECTED EXAMPLES}

In this section, we study the cohomology of some special $p$-group $G_{m}$ for $m \geq 1$ and an element $\bar{\xi}_{m} \in H^{2 m}\left(B G_{m}^{\prime} ; \mathbb{Z} / p\right) /(N g)$ which is not detected by elementary abelian $p$-groups. (However, we see $\xi_{m} \notin H_{n r}\left(G_{m}^{\prime} ; \mathbb{Z} / p\right)$ for $m \geq 2$.)

Let $G^{\prime}$ be the special $p$-group of exponent $p$ defined by

$$
\begin{aligned}
0 & \rightarrow\left\langle c, c_{3}, c_{4}\right\rangle \rightarrow G^{\prime} \rightarrow\left\langle a_{1}, a_{2}, a_{3}, a_{4}\right\rangle \rightarrow 0 \\
\text { with } \quad\left[a_{1}, a_{2}\right] & =\left[a_{3}, a_{4}\right]=c, \quad\left[a_{1}, a_{3}\right]=c_{3}, \quad \text { and } \quad\left[a_{2}, a_{4}\right]=c_{4},
\end{aligned}
$$

and the other pairs commute. (Such a group exists by the reason similar to that for $G s$ stated in $\S 11$. ) The difference between the definitions of $G s$ and $G^{\prime}$ is just $\left[a_{i}, a_{4}\right]=c_{4}$ for $i=1$ or 2 . However, note that $G^{\prime}$ has the rank 2 elementary abelian $p$-subgroup

$$
\left\langle a_{1} a_{3}^{-1}, a_{2} a_{4}^{-1}\right\rangle \cong \mathbb{Z} / p \oplus \mathbb{Z} / p
$$

while $G s$ does not.

Let $G_{m}^{\prime}$ be the central product of $(m-1)$-th copies of $G^{\prime}$ and one $G s$ :

$$
G_{m}^{\prime}=G s \cdot G^{\prime} \cdot \ldots \cdot G^{\prime}=G s \times\langle c\rangle G^{\prime} \times\langle c\rangle \ldots \times\langle c\rangle G^{\prime},
$$

which is generated by $a_{1}, \ldots, a_{4 m}, c, c_{3}, c_{4}, c_{7}, \ldots, c_{4 m-1}, c_{4 m}$. The comutators are given for $1 \leq i \leq m$ :

$$
\begin{gathered}
{\left[a_{4 i-3}, a_{4 i-2}\right]=\left[a_{4 i-1}, a_{4 i}\right]=c} \\
{\left[a_{4 i-3}, a_{4 i-1}\right]=c_{4 i-1}, \quad \text { and }\left[a_{4 i-2}, a_{4 i}\right]=c_{4 i} \text { for } i \neq 1,} \\
\text { but }\left[a_{1}, a_{4}\right]=c_{4} .
\end{gathered}
$$

Let us write $a^{\Lambda}=a_{1}^{\lambda_{1}} \ldots a_{4 m}^{\lambda_{4 m}}$ and $\Lambda=\left(\lambda_{1}, \ldots, \lambda_{4 m}\right)$. Note also that $x_{i} \mid\left\langle a^{\Lambda}\right\rangle=$ $\lambda_{i}$. The commutator is given by the definition

$$
\begin{gathered}
{\left[a^{\Lambda}, a^{M}\right]=c^{d} c_{3}^{d_{3}} c_{4}^{d_{4}} \ldots c_{4 m}^{d_{4 m}},} \\
\text { where } d=\sum_{i}\left(\lambda_{4 i-3} \mu_{4 i-2}-\lambda_{4 i-2} \mu_{4 i-3}+\lambda_{4 i-1} \mu_{4 i}-\lambda_{4 i} \mu_{4 i-1}\right), \\
d_{4 i-1}=\lambda_{4 i-3} \mu_{4 i-1}-\lambda_{4 i-1} \mu_{4 i-3}, \quad d_{4 i}=\lambda_{4 i-2} \mu_{4 i}-\lambda_{4 i} \mu_{4 i-2}(i \neq 1), \\
\text { and } d_{4}=\lambda_{1} \mu_{4}-\lambda_{4} \mu_{1} .
\end{gathered}
$$

By the arguments similar to the proof of Lemma 11.1, we have

Lemma 12.1. Let $\left\langle\Lambda_{1}, \ldots, \Lambda_{2 m}\right\rangle$ and $\left\langle\Lambda_{1}^{\prime}, \ldots, \Lambda_{2 m}^{\prime}\right\rangle$ generate the same $2 m$-dimensional vector space in $(\mathbb{Z} / p)^{4 m}$. Then $\left\langle a^{\Lambda_{1}}, \ldots, a^{\Lambda_{2 m}}\right\rangle$ is an abelian group if and only if $\left\langle a^{\Lambda_{1}^{\prime}}, \ldots, a^{\Lambda_{2 m}^{\prime}}\right\rangle$ is as well.

Lemma 12.2. Let $\xi_{m}=x_{1} x_{2} x_{5} x_{6} \ldots x_{4 m-3} x_{4 m-2} \in H^{*}\left(B G_{m}^{\prime} ; \mathbb{Z} / p\right)$. Then the corresponding element $\bar{\xi}_{m} \in H^{*}\left(B G_{n}^{\prime} ; \mathbb{Z} / p\right) /(N g)$ is not detected by elementary abelian p-groups.

Proof. Suppose that $\bar{\xi}_{m}$ is detected by

$$
\left\langle g_{1}, g_{2}, \ldots, g_{2 m}\right\rangle \cong \mathbb{Z} / p \oplus \ldots \oplus \mathbb{Z} / p
$$


such that $\bar{\xi}_{m} \mid\left\langle g_{1}, \ldots, g_{2 m}\right\rangle \neq 0$, and $\bar{x}_{i}\left|\left\langle g_{j_{i}}\right\rangle=x_{i}\right|\left\langle g_{j_{i}}\right\rangle \neq 0$ for some $j_{i}$. By using the preceding lemma, we can take generators adequately so that $g_{i}=a^{\Lambda_{i}}$ with

$$
\begin{gathered}
\Lambda_{1}=(1,0, *, *|0,0, *, *| \ldots \mid 0,0, *, *), \\
\Lambda_{2}=(0,1, *, *|0,0, *, *| \ldots \mid 0,0, *, *), \\
\Lambda_{5}=(0,0, *, *|1,0, *, *| \ldots \mid 0,0, *, *), \\
\Lambda_{6}=(0,0, *, *|0,1, *, *| \ldots \mid 0,0, *, *), \\
\ldots \\
\Lambda_{4 m-3}=(0,0, *, *|0,0, * *| \ldots \mid 1,0, *, *), \\
\Lambda_{4 m-2}=(0,0, *, *|0,0, *, *| \ldots \mid 0,1, *, *) .
\end{gathered}
$$

Let $i>1$. Consider the commutativity of $L=\Lambda_{4 i-3}$ (e.g., $\lambda_{4 i-3}=1$ and $\lambda_{4 i-2}=$ $0)$ and $M=\Lambda_{1}\left(\mu_{4 i-3}=\mu_{4 i-2}=0\right)$. Since $d_{4 i-1}=\lambda_{4 i-3} \mu_{4 i-1}-\lambda_{4 i-1} \mu_{4 i-3}=0$, we see that

$$
\mu_{4 i-1}=0 \quad \text { from } \mu_{4 i-3}=0 \text { and } \lambda_{4 i-3}=1 .
$$

Similarly, we have $\mu_{4 i}=0$ from the commutativity of $\Lambda_{4 i-2}$ and $\Lambda_{1}$. Thus we see that

$$
\Lambda_{1}=(1,0, *, *|0,0,0,0| \ldots \mid 0,0,0,0) .
$$

We also have $\Lambda_{2}=(0,1, *, *|0,0,0,0| \ldots \mid 0,0,0,0)$. Let $\Lambda=\Lambda_{1}$ and $M=\Lambda_{2}$. By the commutativity and the facts $d_{3}=d_{4}=0$, we see that $\mu_{3}=\mu_{4}=0$; that is,

$$
\Lambda_{2}=(0,1,0,0|0,0,0,0| \ldots \mid 0,0,0,0) .
$$

However, this is a contradiction. Indeed,

$$
d=\sum_{i}\left(\lambda_{4 i-3} \mu_{4 i-2}-\lambda_{4 i-2} \mu_{4 i-3}+\lambda_{4 i-1} \mu_{4 i}-\lambda_{4 i} \mu_{4 i-1}\right)=1 \neq 0 .
$$

Lemma 12.3. We have $\xi_{m} \notin N g\left(G_{m}^{\prime}\right)$. Indeed, $Q_{0} \ldots Q_{2 m-2}\left(\xi_{m}\right) \neq 0$ in $H^{*}\left(B G_{m}^{\prime} ; \mathbb{Z} / p\right)$.

Proof. If $Q_{0} \ldots Q_{2 m-2}\left(\xi_{m}\right) \neq 0$ in $H^{*}\left(B G_{m}^{\prime} ; \mathbb{Z} / p\right)$, then we have $\xi_{m} \notin N g\left(G_{m}^{\prime}\right)$ from Lemma 3.6. So we will prove $Q_{0} \ldots Q_{2 m-2}\left(\xi_{m}\right) \neq 0$. that

We recall that $G_{m}^{\prime}$ is the central product of $G s$ and copies of $G^{\prime}$, which means

$$
0 \rightarrow \bigoplus^{m-1} \mathbb{Z} / p \rightarrow G s \times G^{\prime} \times \ldots \times G^{\prime} \stackrel{p r}{\rightarrow} G_{m}^{\prime} \rightarrow 0 .
$$

We consider the composition map

$$
\begin{aligned}
f^{*}: H^{*}\left(B G_{m}^{\prime} ; \mathbb{Z} / p\right) \stackrel{p r . *}{\rightarrow} H^{*}(B G s ; \mathbb{Z} / p) \otimes H^{*}\left(B G^{\prime} ; \mathbb{Z} / p\right)^{\otimes(m-1)} \\
\quad \rightarrow H=H^{*}(B G s ; \mathbb{Z} / p) \otimes H^{*}\left(B(\mathbb{Z} / p)^{2} ; \mathbb{Z} / p\right)^{\otimes(m-1)} .
\end{aligned}
$$

Here the last map is the restriction map for $(\mathbb{Z} / p)^{2} \subset G^{\prime}$. Indeed, we have the injection $f: G s \oplus(\mathbb{Z} / p)^{2 m-2} \subset G_{m}^{\prime}$.

The above restriction map is explicitly written as

$$
\begin{gathered}
H^{*}\left(B G^{\prime} ; \mathbb{Z} / p\right) \rightarrow H^{*}\left(B\left\langle a_{4 i-3} a_{4 i-1}^{-1}, a_{4 i-2} a_{4 i}^{-1}\right\rangle ; \mathbb{Z} / p\right) \\
\cong \mathbb{Z} / p\left[y_{4 i-3}, y_{4 i-2}\right] \otimes \Lambda\left(x_{4 i-3}, x_{4 i-2}\right),
\end{gathered}
$$

where $y_{4 i-3},-y_{4 i-1} \mapsto y_{4 i-3}$ and $y_{4 i-2},-y_{4 i} \mapsto y_{4 i-2}$. 
We want to show that $f^{*}\left(Q_{0} \ldots Q_{2 m-2}\left(\xi_{m}\right)\right) \neq 0$. For $i>1$, we note that

$$
Q_{2 i-3}\left(x_{4 i-3}\right)=y_{4 i-3}^{p^{2 i-3}}, \quad Q_{2 i-2}\left(x_{4 i-2}\right)=y_{4 i-2}^{p^{2 i-2}} \text {. }
$$

From Lemma 11.2, we also note that

$$
Q_{0}\left(x_{1} x_{2}\right)=y_{1} x_{2}-y_{2} x_{1} \neq 0 \text { in } H^{*}(B G s ; \mathbb{Z} / p) .
$$

Here we give an order for elements in the cohomology

$$
H=H^{*}\left(B\left(G s \oplus(\mathbb{Z} / p)^{2 m-2}\right) ; \mathbb{Z} / p\right) .
$$

We define the lexicographic order $<$ by

$$
y_{5}^{i_{5}} y_{6}^{i_{6}} \ldots y_{4 m-3}^{i_{4 m-3}} y_{4 m-2}^{i_{4 m-2}} x<y_{5}^{j_{5}} y_{6}^{j_{6}} \ldots y_{4 m-3}^{j_{4 m-3}} y_{4 m-2}^{j_{4 m-2}} x^{\prime}
$$

if there is an $s$ such that $i_{s}<j_{s}$ and $i_{k}=j_{k}$ for all $s<k$, where

$$
x, x^{\prime} \in H^{*}(B G s ; \mathbb{Z} / p) \otimes \Lambda\left(x_{5}, x_{6}, \ldots, x_{4 m-3}, x_{4 m-2}\right) .
$$

The operation $Q_{i}$ is a derivation, and we have

$$
\begin{gathered}
f^{*}\left(Q_{0} \ldots Q_{2 m-2}\left(\xi_{m}\right)\right) \\
=Q_{0}\left(x_{1} x_{2}\right) Q_{1}\left(x_{5}\right) Q_{2}\left(x_{6}\right) \ldots Q_{2 m-3}\left(x_{4 m-2}\right) Q_{2 m-2}\left(x_{4 m-2}\right) \bmod (F) \\
=\left(y_{1} x_{2}-y_{2} x_{1}\right) y_{5}^{p} y_{6}^{p^{2}} \ldots y_{4 m-3}^{p^{2 m-3}} y_{4 m-2}^{p^{2 m-2}} \bmod (F)
\end{gathered}
$$

where $(F)$ is the filtration generated by elements of the smaller order rather than the element on the right hand side. In particular, it is non-zero.

Remark. To see $x \in H_{n r}^{*}(G ; \mathbb{Z} / p)$, from the definition of the unramified cohomology by Peyre, we need to check that

$$
\left.\partial_{g}(x)=0 \in H^{*-1}(B H ; \mathbb{Z} / p)\right)
$$

for all $H \oplus \mathbb{Z} / p \cong H \oplus\langle g\rangle \subset G$. When $*>2$ these $H$ need not be abelian. This seems that $x \in H_{n r}^{*}(G ; \mathbb{Z} / p)$ is something of a rare case. Indeed, from the (proof of) the preceding lemma, we see that $G s \oplus(\mathbb{Z} / p)^{2 m-2} \subset G_{m}^{\prime}$ and

$$
\begin{gathered}
\partial_{a_{4 m-2}}\left(\xi_{m}\right)=f^{*}\left(\xi_{m}\right) /\left(x_{4 m-2}\right)=x_{1} x_{2} x_{5} x_{6} \ldots x_{4 m-3} \\
\quad \neq 0 \in H^{2 m-1}\left(B\left(G s \oplus(\mathbb{Z} / p)^{2 m-3}\right) ; \mathbb{Z} / p\right) .
\end{gathered}
$$

So $\xi_{m} \notin H_{n r}^{2 m}\left(G_{m}^{\prime} ; \mathbb{Z} / p\right)$ for $m \geq 2$. (However, we do not yet see $q^{*}\left(\xi_{m}\right) \notin$ $H_{n r}^{*}\left(k\left(W_{*}\right)^{G_{m}^{\prime}} ; \mathbb{Z} / p\right)$.)

Corollary 12.4. There is an element in $H^{2 m}\left(B G_{m}^{\prime} ; \mathbb{Z} / p\right) /(N g)$ which is not detected by abelian subgroup. Namely, the restriction map

$$
\text { Res : } H^{2 m}\left(B G_{m}^{\prime} ; \mathbb{Z} / p\right) /(N g) \rightarrow \Pi_{A ; \text { abelian }} H^{2 m}(B A ; \mathbb{Z} / p) /(N g)
$$

is not injective.

\section{ACKNOWLEDGMENTS}

The second author learned theories of cohomological invariants from discussions with Burt Totaro and Patrick Brosnan. Discussions with Alexander Vishik, Zinovy Reichstein, Kirill Zainoulline, Nikita Semenov, Masaharu Kaneda, Charles Vial and Dinesh Deshpande were very helpful in the writing of this paper. The referee corrected many errors in the first versions of this paper, and the authors thank them very much. 


\section{REFERENCES}

[Ad-Mi] A. Adem and J. Milgram. Cohomology of finte groups. Grund. Math. Wissenschaften Vol. 309, Springer-Verlag (2004) x+327 pp. MR2035696 (2004k:20109)

[Be] K. J. Becher. Virtuelle Formen. In Mathematische Institute, Georg-August-Universit Gottingen:Seminor 2003-2004. 143-150. MR2181575 (2006g:11079)

[Ben] D. Benson. Representations and cohomology. II: Cohomology of groups and modules. Cambridge Studies in Advanced Mathematics 31 (1991) x+278 pp. MR1156302 (93g:20099)

[Bl-Og] S. Bloch and A. Ogus. Gersten's conjecture and the homology of schemes. Ann. Scient. Éc. Norm. Sup. 7 (1974), 181-202. MR0412191 (54:318)

[Bo] F. Bogomolov. The Brauer group of quotient spaces by linear group actions. Math. USSR Izvestiya 30 (1988), 455-485. MR903621 (88m:16006)

[Bo-Pe-Ts] F. Bogomolov, T. Petrov and Y. Tschinkel. Unramified cohomology of finite groups of Lie type. 55-73, Progr. Math., 282, Birkhauser Boston Inc., Boston, MA, 2010. MR.2605165

[Bor] A. Borel. Sur la cohomologie des espaces fibres principaux et des espaces homogenes de groupes de Lie compacts. Ann. Math. 57 (1953), 115-207. MR0051508 (14:490e)

[Br-Re-Vi] P. Brosnan. Z. Reichstein and A. Vistoli. Essential dimension, spinor groups, and quadratic forms. Ann. Math. (2) $\mathbf{1 7 1}$ (2010), no. 1, 553-554. MR2537108 (2010i:14014)

[Co] J. L. Colliot-Thelene. Cycles algebraiques de torsion et $K$-theorie algebrique. In: Arithmetic algebraic geometry (Tronto, 1991). Lec. Note Math. Springer, Berlin 1553 (1993), 1-49. MR.1338859 (96h:14006)

[Ga-Me-Se] S. Garibaldi, A. Merkurjev and J. P. Serre. Cohomological invariants in Galois cohomology. University Lect. Series Vol. 28, AMS (2003). MR1999383(2004f:11034)

[Gu1] P. Guillot. The Chow ring of $G_{2}$ and Spin(7). J. Reine Angew. Math. 604 (2007), 137-158. MR2320315 (2008e:14005)

[Gu2] P. Guillot. Geometric methods for cohomological invariants. Document Math. 12 (2007), 521-545. MR2365912 (2008m:14015)

[Kah] B. Kahn. Classes de Stiefel-Whitney de formes quadratiques et representations galoisiennes reelles. Invent. Math. 78 (1984), 223-256. MR767193 (86g:12011)

[Ka] M. Kameko. Poincaré series of cotorsion products. Preprint (2005).

[Ka-Mi] M. Kameko and M. Mimura. Mùi invariants and Milnor operations, Geometry and Topology Monographs 11 (2007), 107-140. MR2402803 (2009g:55020)

[Ka-Ya] M. Kameko and N. Yagita. The Brown-Peterson cohomology of the classifying spaces of the projective unitary groups $P U(p)$ and exceptional Lie group Trans. of A. M. S. 360 (2008), 2265-2284. MR2373313 (2008m:55008)

[Ko-Ya] A. Kono and N. Yagita. Brown-Peterson and ordinary cohomology theories of classifying spaces for compact Lie groups. Trans. of A. M. S. 339 (1993), 781-798. MR1139493 (93m:55006)

[Ma-Mi] I. Madsen and J. Milgram. The classifying spaces for surgery and cobordism of manifolds. Ann of Math. Studies, Princeton Univ. Press 92 (1979). MR548575 (81b:57014)

[Mi] J. Milnor. Algebraic K-theory and quadratic forms. Invent. Math. 9 (1970), 318-344. MR0260844(41:5465)

[Mo-Vi] L. Molina and A. Vistoli. On the Chow rings of classifying spaces for classical groups. Rend. Sem. Mat. Univ. Padova 116 (2006), 271-298. MR.2287351 (2007k:14006)

[Or-Vi-Vo] D. Orlov,A. Vishik and V. Voevodsky. An exact sequence for Milnor's K-theory with applications to quadratic forms. Ann. of Math. 165 (2007) 1-13. MR2276765 (2008c:19001)

[Pa] W. Paranjape. Some spectral sequences for filtered complexes and applications. J. Algebra 186 (1996) 793-806. MR1424593 (97h:55022)

[Pe] E. Peyre. Unramified cohomology of degree 3 and Noether's problem. Invent. Math. 171 (2008), 191-225. MR 2358059 (2008m:12011)

[Qu] D. Quillen. The mod 2 cohomology rings of extra-special 2-groups and the spinor groups. Math. Ann. 194 (1971), 197-212. MR0290401(44:7582)

[Re] Z. Reichstein. On the notion of essential dimension for algebraic groups. Transform. groups 5 (2000), 265-304. MR.1780933 (2001j:20073) 
[Ro] M. Rost. On the basic correspondence of a splitting variety. Preprint (2006)

[Sa] D. Saltman. Noether's problem over an algebraically closed field. Invent. Math. $\mathbf{7 7}$ (1984), 71-84. MR751131 (85m:13006)

[Su-Jo] A. Suslin and S. Joukhovistski. Norm Variety. J. Pure and Appl. Algebra 206 (2006) 245-276. MR 2220090 (2008a:14015)

[Suz] M. Suzuki. Group theory II. Grund. Math. Wissenschaften Vol. 17, Springer-Verlag (1986) x+621 pp. MR815926(87e:20001)

[Te-Ya] M. Tezuka and N. Yagita. The varieties of the mod $p$ cohomology rings of extraspecial p-groups for an odd prime p. Math. Proc. Cambridge Phil. Soc. 94 (1983) 449-459. MR720796 (85g:20069)

[Tod] H. Toda. Cohomology mod 3 of the classifying space $B F_{4}$ of the exceptional group F4. J. Math. Kyoto Univ. 13 (1973) 97-115. MR0321086 (47:9619)

[To] B. Totaro. The Chow ring of classifying spaces. Proc. of Symposia in Pure Math. "Algebraic K-theory" (1997:University of Washington, Seattle) 67 (1999), 248-281. MR1743244 (2001f:14011)

[Vi] A. Vistoli. On the cohomology and the Chow ring of the classifying space of $P G L_{p}$. J. Reine Angew. Math. 610 (2007) 181-227. MR2359886 (2008k:14011)

[Via] C. Vial. Operations in Milnor K-theory. J. Pure Appl. Algebra 213 (2009) 1325-1345. MR:2497581(2010d:19002)

[Vo1] V. Voevodsky. The Milnor conjecture. www. math. uiuc. edu/K-theory/0170 (1996).

[Vo2] V. Voevodsky. Motivic cohomology with $\mathbb{Z} / 2$ coefficient. Publ. Math. IHES 98 (2003), 59-104. MR.2031199 (2005b:14038b)

[Vo3] V. Voevodsky (Noted by Weibel). Voevodsky's Seattle lectures : $K$-theory and motivic cohomology Proc. of Symposia in Pure Math. "Algebraic K-theory" (1997:University of Washington, Seattle) 67 (1999), 283-303. MR.1743245 (2001i:14029)

[Vo4] V. Voevodsky. Reduced power operations in motivic cohomology. Publ. Math. IHES 98 (2003),1-57. MR.2031198 (2005b:14038a)

[Vo5] V. Voevodsky. On motivic cohomology with $\mathbb{Z} / l$-coefficients. www. math. uiuc. edu/Ktheory/0631 (2003).

[Vo6] V. Voevodsky. Motivic Eilenberg MacLane spces. www. math. uiuc. edu/Ktheory/0864 (2007).

[Ya1] N. Yagita. Applications of Atiyah-Hirzebruch spectral sequence for motivic cobordism. Proc. London Math. Soc. 90 (2005) 783-816. MR2137831 (2006d:57049)

[Ya2] N. Yagita. Coniveau filtration of cohomology of groups. Proc. London Math. Soc. 101 (2010), 179-206. MR.2661245

Department of Mathematics, Faculty of Science, Ryukyu University, Okinawa, Japan

E-mail address: tez@sci.u-ryukyu.ac.jp

Department of Mathematics, Faculty of Education, Ibaraki University, Mito, Ibaraki, JAPAN

E-mail address: yagita@mx.ibaraki.ac.jp 